\title{
Dense cores and star formation in the giant molecular cloud Vela $C^{\star, \star \star, \star \star \star}$
}

\author{
F. Massi ${ }^{1}$, A. Weiss ${ }^{2}$, D. Elia ${ }^{3}$, T. Csengeri ${ }^{2}$, E. Schisano ${ }^{3,4}$, T. Giannini ${ }^{5}$, T. Hill ${ }^{6}$, D. Lorenzetti ${ }^{5}$, K. Menten ${ }^{2}$, \\ L. Olmi ${ }^{1}$, F. Schuller ${ }^{2}$, F. Strafella ${ }^{7}$, M. De Luca ${ }^{8}$, F. Motte ${ }^{9}$, and F. Wyrowski ${ }^{2}$ \\ ${ }^{1}$ INAF - Osservatorio Astrofisico di Arcetri, Largo E. Fermi 5, 50125 Firenze, Italy \\ e-mail: fmassi@arcetri.astro.it; olmi@arcetri.astro.it \\ ${ }^{2}$ Max-Planck-Institut für Radioastronomie, Auf dem Hügel 69, 53121 Bonn, Germany \\ e-mail: aweiss@mpifr-bonn.mpg.de \\ ${ }^{3}$ INAF - Istituto di Astrofisica e Planetologia Spaziali, Via Fosso del Cavaliere 100, 00133 Roma, Italy \\ ${ }^{4}$ INAF - Istituto di Radioastronomia, and Italian ALMA Regional Centre, Via P. Gobetti 101, 40129 Bologna, Italy \\ ${ }^{5}$ INAF - Osservatorio Astronomico di Roma, Via Frascati 33, 00078 Monte Porzio Catone, Italy \\ ${ }^{6}$ Atacama Large Millimeter/Submillimeter Array, Joint ALMA Observatory, Alonso de Córdova 3107, Vitacura 763-0355, \\ Santiago, Chile \\ 7 Dipartimento di Matematica e Fisica "Ennio De Giorgi”, Università del Salento, CP 193, 73100 Lecce, Italy \\ ${ }^{8}$ École Normale Supérieure, CNRS, Observatoire de Paris, UMR 8112, LERMA, Paris, France \\ ${ }^{9}$ Université Grenoble Alpes, CNRS, IPAG, 38000 Grenoble, France
}

Received 11 January 2019 / Accepted 5 July 2019

\begin{abstract}
Context. The Vela Molecular Ridge is one of the nearest ( $700 \mathrm{pc}$ ) giant molecular cloud (GMC) complexes hosting intermediate-mass (up to early B, late O stars) star formation, and is located in the outer Galaxy, inside the Galactic plane. Vela C is one of the GMCs making up the Vela Molecular Ridge, and exhibits both sub-regions of robust and sub-regions of more quiescent star formation activity, with both low- and intermediate(high)-mass star formation in progress.

Aims. We aim to study the individual and global properties of dense dust cores in Vela C, and aim to search for spatial variations in these properties which could be related to different environmental properties and/or evolutionary stages in the various sub-regions of Vela C.

Methods. We mapped the submillimetre $(345 \mathrm{GHz})$ emission from vela C with LABOCA (beam size $\sim 19$.'2, spatial resolution $\sim 0.07 \mathrm{pc}$ at $700 \mathrm{pc}$ ) at the APEX telescope. We used the clump-finding algorithm CuTEx to identify the compact submillimetre sources. We also used SIMBA $(250 \mathrm{GHz})$ observations, and Herschel and WISE ancillary data. The association with WISE red sources allowed the protostellar and starless cores to be separated, whereas the Herschel dataset allowed the dust temperature to be derived for a fraction of cores. The protostellar and starless core mass functions (CMFs) were constructed following two different approaches, achieving a mass completeness limit of $3.7 M_{\odot}$.

Results. We retrieved 549 submillimetre cores, 316 of which are starless and mostly gravitationally bound (therefore prestellar in nature). Both the protostellar and the starless CMFs are consistent with the shape of a Salpeter initial mass function in the high-mass part of the distribution. Clustering of cores at scales of $1-6 \mathrm{pc}$ is also found, hinting at fractionation of magnetised, turbulent gas.
\end{abstract}

Key words. ISM: structure - submillimeter: ISM - ISM: individual objects: Vela Molecular Ridge - stars: formation stars: protostars

\section{Introduction}

It has been known for a long time that the interstellar medium is arranged into a hierarchical distribution of structures, from the

\footnotetext{
* Tables 1 and 2 and the reduced LABOCA image are only available at the CDS via anonymous ftp to cdsarc.u-strasbg.fr (130.79.128.5) or via http://cdsarc.u-strasbg.fr/viz-bin/ qcat? J $/ A+A / 628 / A 110$

$\star \star$ Based on observations made with APEX telescope in Llano de Chajnantor (Chile) under ESO programme ID 089.C-0744 and MPIfR programme ID M-087.F-0043-2011.

$\star \star \star$ Herschel is an ESA space observatory with science instruments provided by European-led Principal Investigator consortia and with important participation from NASA.
}

most massive giant molecular cloud (GMC) complexes down to smaller and less massive entities (namely clouds, clumps, and cores). At the smallest end of the distribution lie dense cores, $\approx 0.1 \mathrm{pc}$ in size with densities of at least $10^{4}-10^{6} \mathrm{~cm}^{-3}$ (see, e.g. Bergin \& Tafalla 2007; Enoch et al. 2007; André et al. 2014). In particular, gravitationally bound starless cores are of the utmost importance, as they are believed to be prestellar in nature and therefore the seeds of stars. In a seminal work, Alves et al. (2007) used near-infrared (NIR) extinction to derive the core mass function $(\mathrm{CMF})$ in the Pipe Nebula $(d \sim 130 \mathrm{pc})$ with a spatial resolution of $0.03 \mathrm{pc}$. These latter authors found that the shape of the CMF is remarkably similar to the stellar initial mass function (IMF; e.g. Kroupa et al. 1993; Scalo 1998; Chabrier 2003) but scaled to higher masses by a factor of about 
three. This is considered to be evidence that the stellar IMF is an outcome of the CMF, provided that an efficiency of $\sim 30 \%$ results from the star-formation processes.

The availability of large bolometer arrays in the submillimetre (submm) and more recently of balloon-borne (the Balloonborne Large Aperture Submillimeter Telescope, BLAST) and space-borne (the Herschel space observatory) far-infrared (FIR) telescopes has allowed the CMF of prestellar cores in the nearest star-forming regions up to Orion to be sampled with high sensitivity and spatial resolution (for a review, see André et al. 2014). These observations have confirmed that the shape of the CMF is consistent with that of the IMF, strengthening the idea of a link between them. It is therefore fundamental to derive CMF and other prestellar core properties in regions with different levels and global efficiency of star formation to check whether the connection between IMF and CMF persists in different environments. In fact, significant differences have recently been found in CMFs derived from observations of farther massive star-forming regions (e.g. Motte et al. 2018), now feasible thanks to the large $\mathrm{mm}$ interferometers. On the other hand, Olmi et al. (2018) used the Hi-Gal catalogue to study the clump mass function in several regions at a range of distances, finding again that its shape generally resembles that of the IMF and suggesting that this supports gravito-turbulent fragmentation of molecular clouds occurring in a top-down cascade.

The Vela Molecular Ridge (VMR) is a GMC complex first mapped in the $\mathrm{CO}(1-0)$ transition (at low spatial resolution) by Murphy \& May (1991), who divided the structure into four main clouds (named A, B, C and D) corresponding to local $\mathrm{CO}(1-$ $0)$ peaks. The VMR is located in the outer Galaxy, inside the Galactic plane, with a kinematic distance of 1-2 kpc. Liseau et al. (1992) analysed all the distance indicators available, concluding that clouds A, C, and D are at $d=700 \pm 200 \mathrm{pc}$. In this regard, we performed a distance test using Gaia DR2 data (Gaia Collaboration 2016, 2018), which is described in Appendix A. We found indications of a value of $950 \mathrm{pc}$ (with an estimated error of $\pm 50 \mathrm{pc}$ ). Since this does not significantly affect our main results, pending further refinements of the distance determination, we will adopt the value of $700 \mathrm{pc}$ for consistency with all previous works. However, we detail the effects of the larger value in the appendix.

Subsequent observational studies have demonstrated that the VMR hosts low- and intermediate-mass (up to early $\mathrm{B}$, late $\mathrm{O}$ stars) star formation in a number of stellar clusters (Liseau et al. 1992; Lorenzetti et al. 1993; Massi et al. 1999, 2000, 2003). It is therefore one of the nearest regions with intermediate-mass star formation in progress, just slightly more distant than Orion. However, unlike Orion, the VMR is located in the Galactic plane, and is therefore probably more representative of typical Galactic molecular cloud complexes. In this respect, sampling the $\mathrm{CMF}$ in the VMR is valuable, since this region makes up a different environment from those already studied in the solar neighbourhood.

The first large mm continuum mapping of the VMR was carried out by Massi et al. (2007) towards Vela D at $250 \mathrm{GHz}$ with SIMBA at the SEST telescope, with a spatial resolution of $\sim 0.08 \mathrm{pc}$, a little less than the size of the largest dense cores. The VMR was then mapped in the FIR by Netterfield et al. (2009) with BLAST. Subsequently, Olmi et al. (2009) combined the data from Massi et al. (2007) and from Netterfield et al. (2009), obtaining a CMF for Vela D whose shape is in good agreement with that of a standard IMF. Hill et al. (2011) studied Vela C using Herschel PACS and SPIRE FIR/submm observations at $70,160,250,350$, and $500 \mu \mathrm{m}$, finding a filamentary structure. They also identified five main sub-regions (see Fig. 1) exhibiting different morphologies (ridges and nests of filaments). Giannini et al. (2012) used the same data to retrieve dense cores and derive a CMF. They identified starless cores based on the lack of emission at $70 \mu \mathrm{m}$ and obtained a prestellar CMF whose high-mass-end is flatter but still consistent with a standard IMF.

The Herschel PACS and SPIRE bands span the emission peak of cold dust, making these data very useful for deriving the dust temperature. On the other hand, $\mathrm{mm}$ and submm continuum emission from dust is more suitable for deriving column densities (and hence masses), since this occurs in the optically thin regime. In addition, ground-based mm observations allow a better angular resolution than space-borne instruments. Therefore, a better way to sample the CMF is by combining FIR (dust temperature) and submm (column density) maps with comparable spatial resolution. In this paper, we report on submm $(345 \mathrm{GHz})$ observations of Vela $\mathrm{C}$ obtained with the Large Apex Bolometer Camera (LABOCA) at the Atacama Pathfinder EXperiment (APEX) telescope (see Fig. 1 for the final LABOCA map), with a spatial resolution of $\sim 19^{\prime \prime}(\sim 0.07 \mathrm{pc}$ at $700 \mathrm{pc})$. By combining ancillary Herschel and Wide-field Infrared Survey Explorer (WISE) data, we have revised the CMF of Vela $\mathrm{C}$ obtained by Giannini et al. (2012). After outlining some well-studied specific regions of Vela $\mathrm{C}$ in Sect. 2, we describe our observations, the sets of ancillary data, and the clump-finding algorithms used in Sect. 3. Our results are presented in Sect. 4 and discussed in Sect. 5. Finally, we summarise our work in Sect. 6.

\section{Young embedded star clusters and HII regions towards Vela C}

Several individual star-forming regions in the VMR have been studied in detail by a number of authors. Three of these regions are relevant for Vela $\mathrm{C}$ and are described in the following.

\section{1. $R C W 34$}

The location of the HII region RCW 34 is enclosed within a box in the map of submm emission from Vela $\mathrm{C}$ shown in Fig. 1. Figure 2 displays an IRAC/Spitzer image of the region at $3.6 \mu \mathrm{m}$ (from the GLIMPSE survey; Benjamin et al. 2003; Churchwell et al. 2009), overlaid with contours of the submm emission. The Spitzer image outlines PAH emission excited by the ionising radiation from the massive stars. Clearly, the submm emission is strictly related to the NIR diffuse emission, confirming that most of the submm structures in the field displayed in Fig. 2 are associated with RCW 34. There are indications that RCW 34 is farther away than Vela C; for example, the total radio flux listed in the Parkes-MIT-NRAO catalogue at $4.85 \mathrm{GHz}$ (Griffith \& Wright 1993 ) is $2.854 \mathrm{Jy}$ (source PMNJ0856-4305), about a factor ten fainter than that of the other HII region, RCW 36 (see below). The $V_{\mathrm{LSR}}$ of the main molecular clump is $\sim 5.5 \mathrm{~km} \mathrm{~s}^{-1}$, as measured in the CS(2-1) transition (Bronfman et al. 1996). On the other hand, the $V_{\text {LSR }}$ of the $\mathrm{C}^{18} \mathrm{O}(1-0)$ clumps of Yamaguchi et al. (1999) are $\sim 7 \mathrm{~km} \mathrm{~s}^{-1}$ in the northern and central parts of Vela $\mathrm{C}$ and only in the southern part are in the range $5-6 \mathrm{~km} \mathrm{~s}^{-1}$. Bik et al. (2010) revised the distance of RCW 34 to $2.5 \mathrm{kpc}$, which confirms that this is a background region.

\section{2. $R C W 36$}

The HII region RCW 36 is one of the most prominent features of Vela C. As shown in Fig. 1, it is situated in the centre of a 


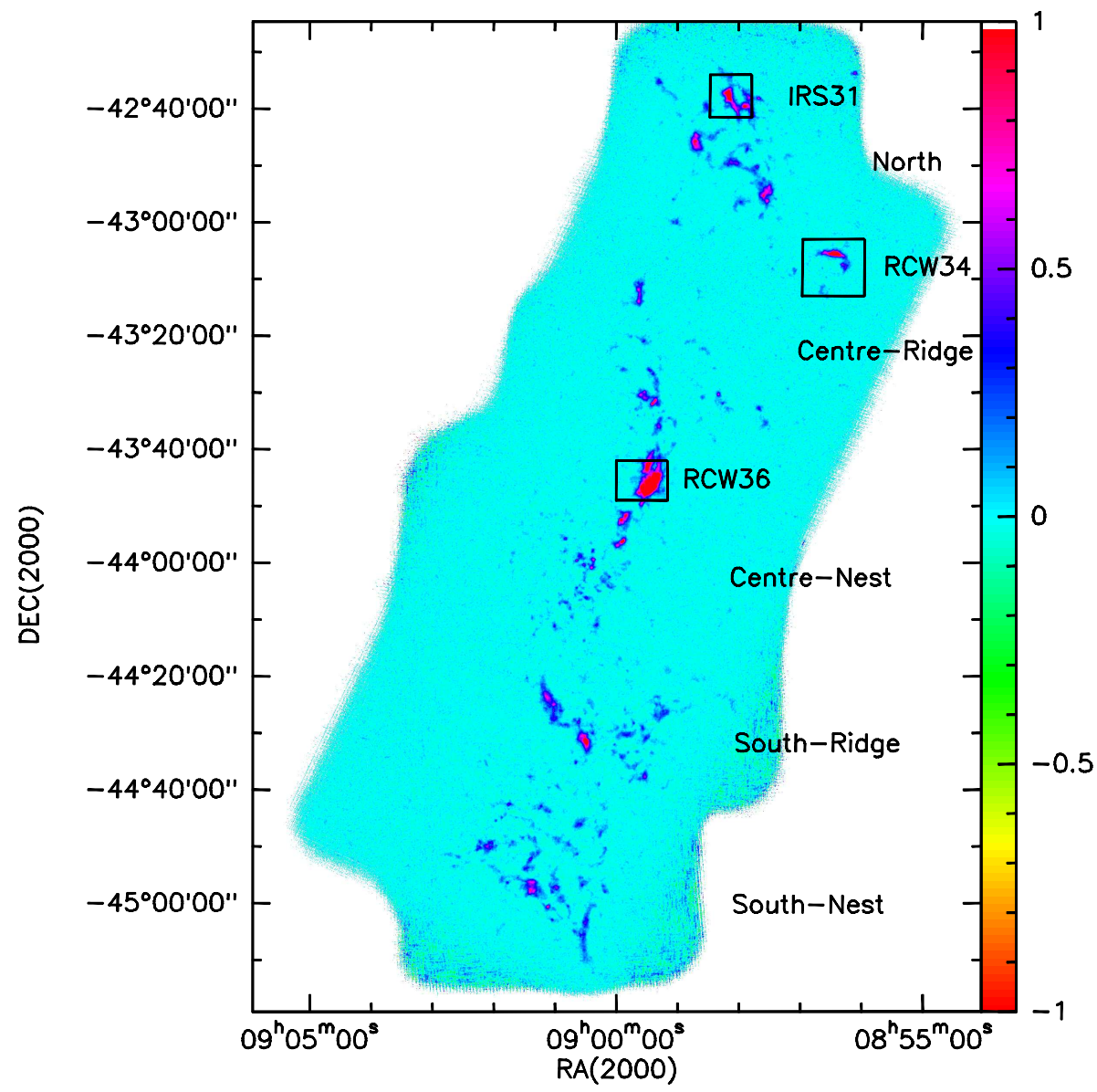

Fig. 1. LABOCA map of $345 \mathrm{GHz}$ emission towards Vela C. The colour scale is indicated in the bar on the right in units of $\mathrm{Jy} \mathrm{beam}^{-1}$. The locations of the HII regions RCW 34 and RCW 36, and the young cluster IRS 31 are enclosed with boxes and labelled (see Figs. 2, 3 , and 4, respectively, for zoomed-in maps of these regions). The sub-regions defined by Hill et al. (2011) are also labelled.

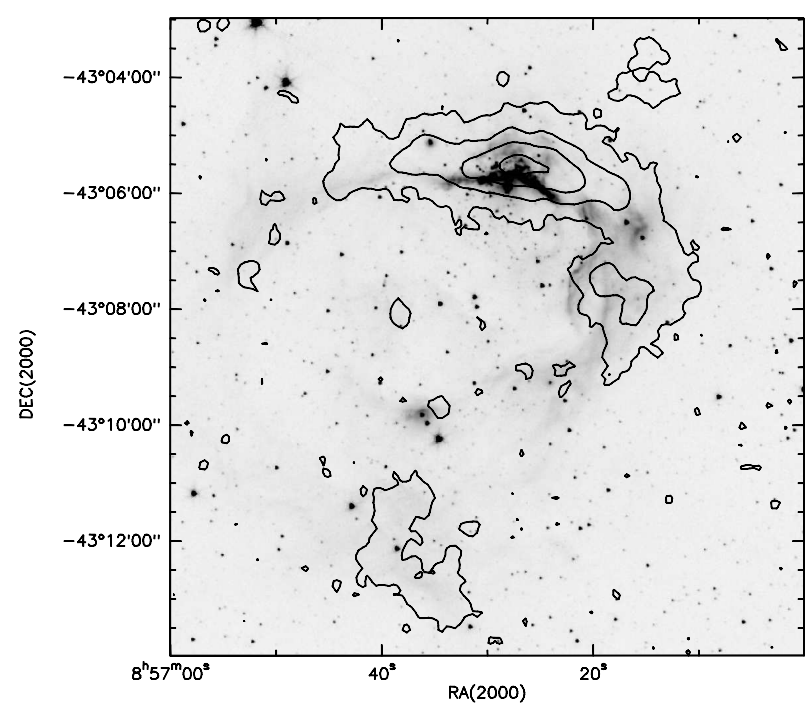

Fig. 2. IRAC/Spitzer image at $3.6 \mu \mathrm{m}$ of the HII region RCW 34, outlining PAH emission, overlaid with contours of submm emission measured with LABOCA. Contours are 0.06, 0.36, 1, 3, and $6 \mathrm{Jy} \mathrm{beam}^{-1}$.

large dust ridge and exhibits the most intense sources of submm emission in the field. This region hosts a young star cluster and the IR source IRS 34 studied by Massi et al. (2003). The ionising sources are \# 68 and 59 of Massi et al. (2003), which were identified by those authors as two late O stars. Subsequently, Ellerbroek et al. (2013) using optical-NIR spectroscopy confirmed that source \#68 (their \#1) is an 08.5-9.5 V star and
\#59 (their \#3) is an O9.5-B0 V star. As shown in Fig. 3, the submm emission is mostly arranged along a ridge, off-centred with respect to the cluster and the ionising stars. Minier et al. (2013) suggest that this actually marks an expanding ring of dust and gas surrounding the cluster that is tilted compared to the line of sight. The Parkes-MIT-NRAO catalogue at $4.85 \mathrm{GHz}$ (Griffith \& Wright 1993) lists a total flux density of 22 Jy from the HII region (source PMNJ0859-4345).

\subsection{IRS 31}

IRS 31 is a young embedded cluster discovered by Massi et al. (2003). It is located in the northern part of Vela C (see Fig. 1) and, as can be seen in Fig. 4, is associated with a strong submm source. Little is known about this cluster, whose most massive member is apparently a Class I source of intermediate mass (Massi et al. 2003). The NIR counterpart of this object (\#14 of Massi et al. 2003) lies 30" east of the submm peak. The dust emission peak appears off-centred compared to the star cluster, as in the RCW 36 region. No radio emission at $4.85 \mathrm{GHz}$ was detected by the Parkes-MIT-NRAO survey.

\section{Observations and data reduction}

\section{1. $\angle A B O C A$}

The observations ${ }^{1}$ were carried out in August 2011, May 2012, and August 2012, with the LABOCA camera (Siringo et al. 2009) at the APEX telescope (Güsten et al. 2006). LABOCA is

1 Project ID: 087.F-0043 (MPIfR), 089.C-0744 (ESO). 


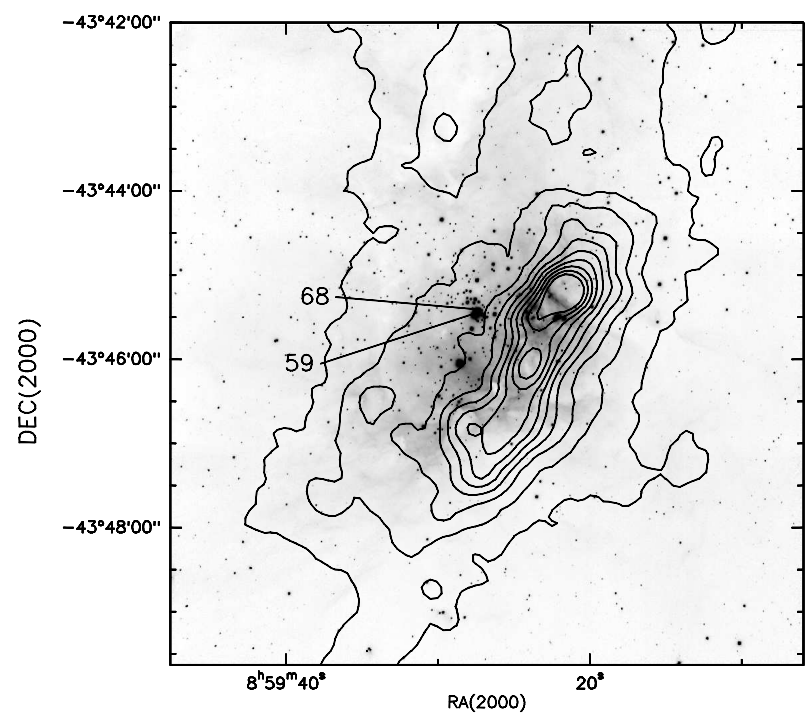

Fig. 3. $K_{s}$ SofI image of the HII region RCW 36, overlaid with contours of submm emission mapped with LABOCA. Contours are in steps of $0.6 \mathrm{Jy}$ beam $^{-1}$ from $0.2 \mathrm{Jy}_{\text {beam }}{ }^{-1}$. The two late $\mathrm{O}$ stars that ionise the gas are labelled using the designation by Massi et al. (2003).

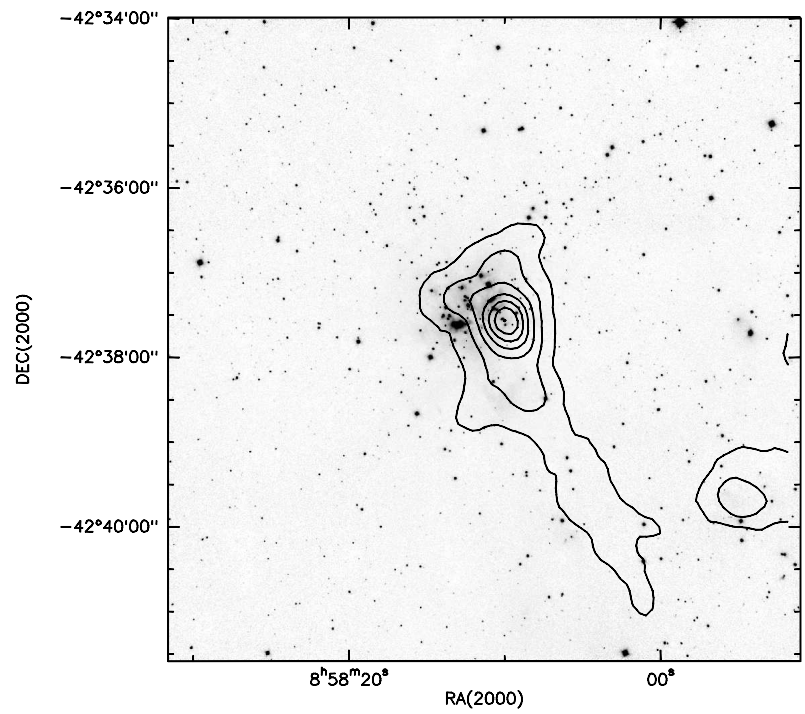

Fig. 4. $K_{s}$ SofI image of the young embedded cluster IRS 31, overlaid with contours of submm emission mapped with LABOCA. Contours are in steps of $0.5 \mathrm{Jy}^{\text {beam }}{ }^{-1}$ from $0.5 \mathrm{Jy} \mathrm{beam}^{-1}$.

an array of 295 bolometers operating at $345 \mathrm{GHz}(0.87 \mathrm{~mm})$ with a beam HPBW of 19.'2. The first set of observations (August $2011)$ is composed of small $\left(\sim 15^{\prime} \times 15^{\prime}\right)$ on-the-fly maps covering the Vela $\mathrm{C}$ cloud, while the second set (May and August 2012) were obtained using single large maps in orthogonal coverages. Pointing and focus were checked regularly during the observations; standard sources such as planets have been used as primary flux calibrators.

The data were reduced using the $B o A$ software $^{2}$ (Schuller 2012), following the steps described in detail by Schuller et al. (2009). The two datasets were then stitched together during the data reduction, and emission from larger scales was iteratively recovered until convergence was reached after four iterations. We

2 http://www.eso.org/sci/activities/apexsv/labocasv. html checked that the source fluxes in the two datasets, when reduced independently, are consistent with each other within a $30 \%$ level.

The final map was obtained using a pixel size of 4". 6 after smoothing with a 9" FWHM Gaussian kernel. This covers the whole Vela C cloud as originally defined in Murphy \& May (1991) and subsequently imaged with Herschel by Hill et al. (2011). The rms typically ranges between 20 and $35 \mathrm{mJy} \mathrm{beam}^{-1}$ over the area, with the largest values obviously found at the map edge. Figure 1 shows the final dust emission distribution. Our observations clearly retrieved the structures already found through Herschel observations (Hill et al. 2011). For the sake of comparison, the sub-regions defined by Hill et al. (2011) are labelled in the figure, along with the two HII regions RCW 34 and RCW 36, and the young embedded cluster IRS 31.

\subsection{SIMBA}

Two regions in Vela $C$ hosting young embedded clusters, IRS 31 and RCW 36 (Massi et al. 2003), were mapped in the $250 \mathrm{GHz}$ (1.2 mm) continuum on May 26, 2003, using the 37-channel SEST IMager Bolometer Array (SIMBA; Nyman et al. 2001) at the Swedish-ESO Submillimetre Telescope (SEST) located at La Silla, Chile. The observations were made at the end of ESO programme 71.C-0088, whose objective was a large-scale map of Vela D (Massi et al. 2007). Two small maps of $600^{\prime \prime} \times 400^{\prime \prime}$ (azimuth $\times$ elevation) were obtained for both regions in fast scanning mode, with a scanning speed of $80^{\prime \prime} \mathrm{s}^{-1}$. The scan towards IRS 31 was repeated three times. The beam HPBW is $24^{\prime \prime}$ and the map rms is $\sim 30 \mathrm{mJy}^{\text {beam }}{ }^{-1}$ for RCW 36 and $\sim 20 \mathrm{mJy} \mathrm{beam}^{-1}$ for IRS 31. Details on the data reduction and calibration steps are given in Massi et al. (2007). These authors also found that their flux calibration is likely to be accurate within $\sim 20 \%$.

\subsection{Clump-finding algorithms}

To obtain a census of the dense core population of Vela $C$, we used the algorithm CuTEx (Molinari et al. 2011), developed to work on FIR/submm maps and widely tested on Herschel maps. CuTEx computes the second derivative of the image, which is related to the curvature of the shape of the brightness distribution. The algorithm then searches for the strongest variations in brightness, which are expected to be at the centre of compact sources. Finally, all the pixels above a threshold limit are identified belonging to a candidate source. We tested different thresholds and selected the highest one which still retrieves all the features distinguishable by visual inspection. This choice minimises the number of artifacts. To strengthen our results we also discarded all the detections where the measured source peak brightness is smaller than three times the brightness rms around the source considered.

We chose CuTEx on the grounds that it is more focused on detecting compact sources compared to other algorithms (e.g. CLUMPFIND) that generally split the (relevant) brightness distribution into different, separated objects. Dust structures $\approx 0.1 \mathrm{pc}$ in size are usually referred to in the literature as "cores". At a distance of $700 \mathrm{pc}$, this spatial scale corresponds to angular sizes $\approx 30^{\prime \prime}$, resolved by LABOCA. Therefore, hereafter we refer to the retrieved compact sources as submm cores. We found 549 Vela C sources and a further 15 submm sources associated with RCW 34 (see Table 1). As shown in Fig. 5, most of the sources exhibit a signal-to-noise ratio ( $\mathrm{S} / \mathrm{N}$; in source flux density) much larger than 3. In particular, the histogram of flux densities, also shown in this figure, exhibits an increase in the number of sources with decreasing flux density down to $F_{\text {compl }} \sim 0.3 \mathrm{Jy}$. This value 


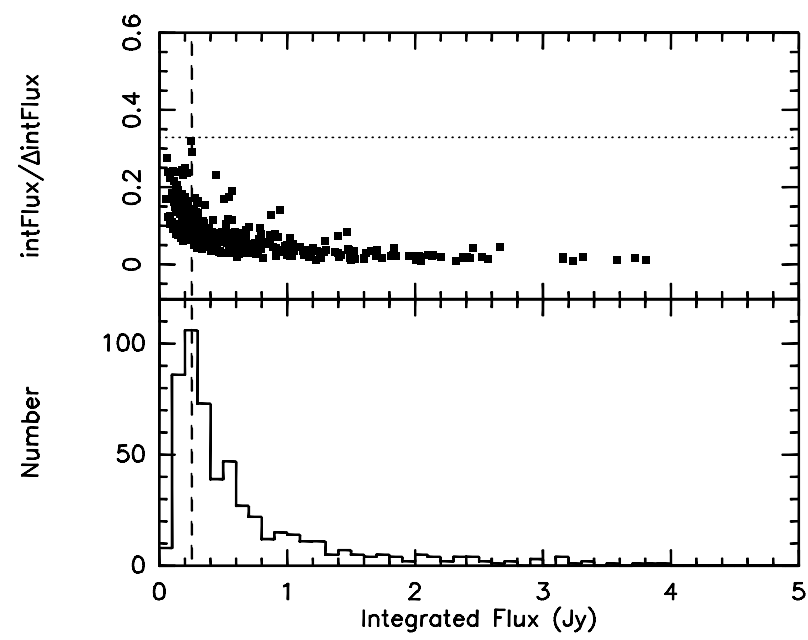

Fig. 5. Top: ratio of flux density to flux density error vs. flux density for all submm sources found with CuTEx; datapoints below the horizontal dotted line have $S / N>3$. Bottom: flux density for all submm sources found with CuTEx. The vertical dashed line marks the estimated completeness limit.

corresponds to sources with $\mathrm{S} / \mathrm{Ns}$ of $\sim 5$ and can be interpreted as our completeness limit for core detection, since the number of sources quickly decreases for flux densities $<0.3 \mathrm{Jy}$. For this reason, we consider only sources above the completeness limit to derive the physical properties of the submm cores.

To allow comparison with other studies, we also tested the algorithm CLUMPFIND (Williams et al. 1994), which was run by Massi et al. (2007) on their SIMBA map (250 GHz) of Vela D. We used a lowest contour level of $\sim 3 \mathrm{rms}$ and an intensity level increment of $\sim 5 \mathrm{rms}$ as input parameters. We performed several runs with different input parameters and found that this choice yielded the best efficiency in terms of source detection based on a visual comparison of the results. We retrieved 291 Vela C submm sources and 7 other submm sources towards RCW 34 (see Table 2). Following Massi et al. (2007), we discarded another 104 CLUMPFIND detections with angular sizes (not deconvolved) smaller than the APEX beam size (see Table 2). However, similar detections in Vela D were classified as real faint sources by De Luca et al. (2007) based on a cross-correlation of databases spanning a wide range of wavelengths.

A visual comparison of the CUTEx and CLUMPFIND outputs shows that most of the 291 CLUMPFIND sources are associated with one or more CuTEx source. There are only a few unmatched CLUMPFIND sources, but a significant number of unmatched CuTEx sources. Interestingly, many of these unmatched CuTEx sources nevertheless are associated with CLUMPFIND sources from the list of the 104 "unresolved" sources, confirming that the latter are mostly real submm cores. We therefore list the 104 "unresolved" submm sources in Table 2 as well. In the end, only a few CLUMPFIND and CuTEx sources do not match; in this respect the two algorithms yield highly consistent results.

The estimated completeness limit $F_{\text {compl }} \sim 0.3$ Jy translates into a mass completeness limit (Eq. (8) of Elia \& Pezzuto 2016, who use the formalism of Hildebrand 1983) of $\sim 1.3 M_{\odot}$, by adopting a dust temperature $T_{\text {dust }}=15 \mathrm{~K}$, a dust opacity $k_{v}=1.85 \mathrm{~g}^{-1} \mathrm{~cm}^{2}$, and a dust-to-gas ratio of 100 (Schuller et al. 2009). However, there are large uncertainties in the dust opacity, which may vary by more than a factor of two. In fact, if we assume $k_{v}=0.5 \mathrm{~g}^{-1} \mathrm{~cm}^{2}$ at $250 \mathrm{GHz}$, as in Massi et al. (2007)
Table 3. Names and definitions used in the text for power-law indices.

\begin{tabular}{lll}
\hline \hline Name & Definition & Astrophysical quantity \\
\hline$\alpha$ & $\alpha=\mathrm{d} \log (\psi) / \mathrm{d} \log (M)$ & $\psi=\mathrm{d} N / \mathrm{d} M$ initial mass function \\
$\alpha^{\prime}$ & $\alpha^{\prime}=\mathrm{d} \log \left(\psi^{\prime}\right) / \mathrm{d} \log (M)$ & $\psi^{\prime}=\mathrm{d} N / \mathrm{d} \log (M)$ initial mass function \\
$\beta$ & $\tau_{v}=\left(\nu / v_{0}\right)^{\beta}$ & $\tau_{\nu}$ dust column density \\
$\gamma$ & $\gamma=\mathrm{d} \log \left(\lambda F_{\lambda}\right) / \mathrm{d} \log (\lambda)$ & $\lambda F_{\lambda}$ spectral energy distribution \\
\hline
\end{tabular}

and in early seminal works like Motte et al. (1998), and a dust emissivity index (see Table 3 for the power-law index names and definitions adopted in this paper) $\beta=2$ (Olmi et al. 2009), we determine $k_{v}=0.86 \mathrm{~g}^{-1} \mathrm{~cm}^{2}$ at $345 \mathrm{GHz}$ (from Eq. (7) of Elia \& Pezzuto 2016). Adopting this value and $T_{\text {dust }}=12.5 \mathrm{~K}$ (in accordance with the discussion in Sect. 4.4), the mass completeness limit increases to $\sim 3.7 M_{\odot}$. As a sidenote, one should be aware that this is a conservative choice: Martin et al. (2012) reviewed the values of opacity in the literature and the trend they found is clearly towards using a $k_{v}$ that is roughly a factor of two larger than that derived from the value used by Massi et al. (2007).

\subsection{Ancillary data: WISE}

Giannini et al. (2012) analysed Herschel observations of Vela $\mathrm{C}$ in the framework of the guaranteed time key programme "HOBYS" (Motte et al. 2010). Using CuTEx, these authors detected a number of compact sources, selecting a robust subsample of 256 objects including both protostellar and starless cores. They identified starless cores out of their sample based on the lack of emission at $70 \mu \mathrm{m}$ and/or of associated IRAS/MSX/Akari IR sources. In this work, we alternatively exploit the association of CuTEx compact sources with IR sources from the WISE all-sky data release (Wright et al. 2010). This provides point-source photometry at 3.6, 4.6, 12, and $22 \mu \mathrm{m}$ with better sensitivity and better angular resolution than IRAS, MSX, and Akari. The latter ranges from $\sim 6^{\prime \prime}$ at $3.6 \mu \mathrm{m}$ to $\sim 12^{\prime \prime}$ at $22 \mu \mathrm{m}$. We retrieved all point sources from the WISE catalogue that are projected towards the same sky area as Vela $\mathrm{C}$, with valid detections either in at least three bands or at $22 \mu \mathrm{m}$, and with photometric errors $<0.3 \mathrm{mag}$ either in all detected bands or at least at $22 \mu \mathrm{m}$. Having valid detections in at least three bands allows one to discriminate between field stars and real young stellar objects based on their colours (see below). On the other hand, heavily embedded young sources might only be detected at $22 \mu \mathrm{m}$ due to their rising spectral energy distribution (SED). First, we discarded all contaminants (i.e. extragalactic objects, PAH emission, shock emission) by following the colour criteria of Koenig et al. (2012), from the sources with detections in every band needed to apply those criteria. This means that all the sources lacking detection in any of those bands are retained. We then selected all remaining entries with the colours of Class I sources, again following Koenig et al. (2012), provided that all the needed colours are available from the WISE photometry. For sources lacking detections in any of the bands required to apply the colour criteria, we computed their spectral index $\gamma$ (see Table 3 for definition) and selected the ones with $\gamma>-0.3$ (Strafella et al. 2015). Sources detected only at $22 \mu \mathrm{m}$ with a photometric error $<0.3 \mathrm{mag}$ were also retained. Out of the 448 WISE Class I sources, only 22 were classified based on their spectral index $\gamma$, and even less (four) are only detected at $22 \mu \mathrm{m}$ with photometric error $<0.3 \mathrm{mag}$. Finally, we crosscorrelated this subsample of WISE red sources and our CuTEx list of cores, considering valid associations to be those where 
the WISE uncertainty ellipse overlaps the ellipse that has the major and minor FWHMs of a CuTEx output as its semi-major and semi-minor axes. Out of the 549 submm cores (excluding RCW 34), 217 were found to be associated with one or more WISE-selected Class I source and were therefore classified as "protostellar". The remaining 332 submm cores were tentatively labelled as "starless".

One concern about WISE photometry is saturation of bright sources in regions of intense diffuse emission. A look at the archived images shows that only a small area towards RCW 36 is saturated in the two upper bands. This area includes 12 submm sources. We checked the MSX Infrared Point Source Catalogue for IR sources with $\gamma>-0.3$ matching any of the 12 sources in projection. Thus, we found another four cores with associated red MSX sources, which were reclassified as protostellar.

\subsection{Ancillary data: Herschel}

Dust emission at submm wavelengths is optically thin even for large column densities (e.g. Schuller et al. 2009), allowing a straightforward determination of gas mass under the assumption of a gas-to-dust mass ratio. Dust temperature is also needed. The availability of Herschel observations between 70 and $500 \mu \mathrm{m}$, with a beam size $\left(18^{\prime \prime} .1\right.$ at $\left.250 \mu \mathrm{m}\right)$ comparable with the APEX one at $345 \mathrm{GHz}$, provides us with a means of deriving reliable dust temperatures. We decided to cross-correlate the sample of Herschel cores from Giannini et al. (2012) and our CuTExextracted sample to infer dust temperatures for the submm cores.

We retrieved the Herschel maps at 70/160/250/350/500 $\mu \mathrm{m}$ observed in the HOBYS key programme and used CuTEx with the same parameters as in Giannini et al. (2012). The compact sources found in each Herschel map were matched following Giannini et al. (2012) to re-build their catalogue of 1686 entries, most of them with a detection in one or two bands only. A set of routines was provided by Giannini (priv. comm.) to make sure that we could quickly reproduce their results. These sources were then matched to the submm cores retrieved with CuTEx by requiring that a circle of the same diameter as the beam at $250 \mu \mathrm{m}$ and centred at the nominal position of the Herschel source overlaps at least partially with the CuTEx ellipse (with axes of $1 F W H M$ ) of a submm core. In quite a few cases, more Herschel sources were associated with the same submm core; we selected the closest match, discarding the others. The reverse case (multiple submm cores associated with one Herschel source) occurred on a few occasions, and we selected the closest match as well. Ultimately, we found that 219 out of 328 submm starless cores and 185 out of 221 submm protostellar cores are associated with Herschel compact sources, meaning that we were only able to match $74 \%$ of the submm cores to Herschel sources. By overlapping CuTEx ellipses, and IR and submm maps, we found that submm sources without a Herschel counterpart are either part of a multiple association with the same Herschel source, or fall towards 250 and $350 \mu \mathrm{m}$ emission which is probably too extended and hence missed by CuTEx in those maps. A small number of other submm sources without a Herschel match may be artifacts, but we checked that in this case their flux density is fainter than our completeness limit.

Only 388 Herschel sources passing the first three selection criteria adopted by Giannini et al. (2012) were suitable to derive a temperature. Therefore, in the end, the subsample of those Herschel compact sources with a temperature determination and matched to submm cores includes only 197 objects (i.e. $51 \%$ out of 388 Herschel entries). By overlaying the positions of Herschel and submm sources, we found that only 3 out of the 48 protostellar sources of Giannini et al. (2012) do not have a matching submm source. It is therefore the starless Herschel sample of these latter authors that exhibits the higher fraction of unmatched sources. A comparison between the Herschel catalogue and our extraction list reveals that we detected more sources over the filamentary features present in the Herschel maps. This is likely due to the approach adopted when treating the Herschel maps, that is, using a higher threshold value over the bright filamentary features of the clouds to prevent a fragmentation of the emission in an implausibly large number of individual sources.

The lists of identified sources from the Herschel and LABOCA maps are therefore partly different. CuTEx also tends to find larger cores on the submm map, whereas these are often split up into a number of smaller sources on the IR maps. This is due to both the differences (in sensitivity) between the two datasets and the slightly different approaches adopted in defining the parameters for the extraction.

\section{Results}

The derived statistical properties of the population of submm cores in Vela $\mathrm{C}$ are described in the following. The involved physical parameters are also discussed along with the main sources of error.

\subsection{Nature of the submm emission near young star clusters and HII regions}

The nature of the submm sources in the most evolved regions of Vela C, namely the young embedded clusters and HII regions, can be further investigated by comparing our LABOCA data at $345 \mathrm{GHz}$ and SIMBA observations of IRS 31 and RCW 36 at $250 \mathrm{GHz}$. Figure 6 shows the pixel-to-pixel ratio of flux density at 345 and $250 \mathrm{GHz}$ after smoothing of the LABOCA map to the spatial resolution of the SIMBA map (24"). This ratio gives some useful information on the dust spectral index, provided contamination from optically thin free-free emission (which would yield a ratio $\sim 1$ ) is negligible.

Both maps of Fig. 6 show that the flux density ratio is $\sim 2.7$ towards the centre of each emitting clump and increases towards the outer parts (up to 5-6 for RCW 36 and 4-5 for IRS 31). This gives some hints on the spatial variations of dust emissivity index and temperature. In principle, an increase in the ratio towards the outer parts may be a signature of a temperature gradient, with the innermost clump regions being colder. This is plausible, particularly for RCW 36 where the clump is externally exposed to an intense UV field from massive stars.

A ratio of $\sim 2.7$ can be obtained (from Eq. (6) of Elia \& Pezzuto 2016) with a dust emissivity index $\beta$ ranging between 1.3 and 1.9 for $T_{\text {dust }}$ between 10 and $30 \mathrm{~K}$, whereas a ratio of $\sim 4$ requires either $\beta \sim 2.5-3.2$ in the same temperature range or a dust temperature exceeding $100 \mathrm{~K}$ with $\beta \sim 1-2$. Larger values of the flux density ratio $(>5)$ require $\beta>3$. Thus, the innermost emission from both clumps is consistent with $\beta \sim 1-2$, the range of values expected in dust cores (see, e.g. Schnee et al. 2014). This agrees with extrapolating the total Parkes-MITNRAO radio flux density at $4.85 \mathrm{GHz}$ from RCW 36 to $345 \mathrm{GHz}$ in the assumption of optically thin emission, which yields a value that is only $\sim 10 \%$ of the total $345 \mathrm{GHz}$ flux density from a similar area. On the other hand, an increase in the dust temperature of the outermost clump layers can only explain part of the increase in flux density ratio, as this would also need unrealistic $\beta$ values. Thus, systematic effects may be involved. Position errors 

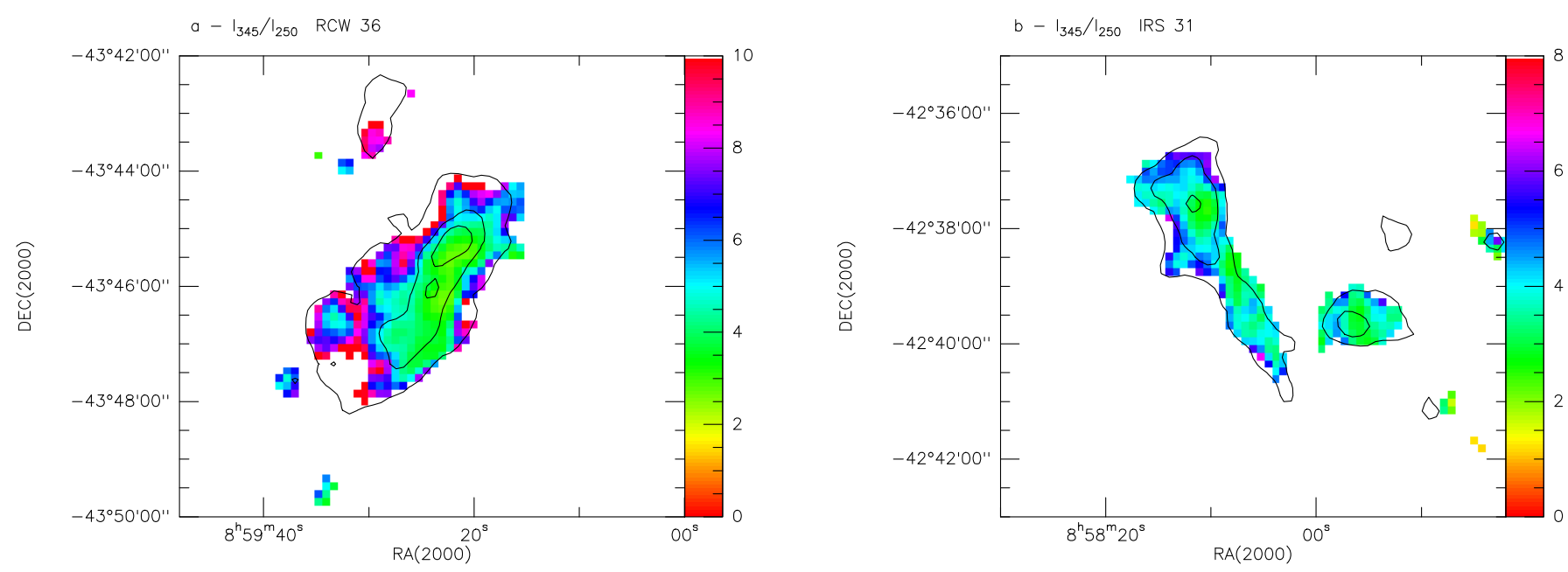

Fig. 6. Colour map of $S_{345} / S_{250}$ towards RCW 36 (panel $a$ ) and IRS 31 (panel $b$ ). The map at $345 \mathrm{GHz}$ (LABOCA) has been smoothed to the angular resolution of the map at $250 \mathrm{GHz}$ (SIMBA) and then divided by it. The emission at $345 \mathrm{GHz}$ is overlaid in contours of 1000,3000 , and $5000 \mathrm{Jy}_{\text {beam }}^{-1}$ (panel $a$ ) and 500,1000 , and $3000 \mathrm{Jy} \mathrm{beam}^{-1}$ (panel $b$ ). Only pixels with a brightness $\sim 5 \mathrm{rms}$ have been selected.

(few arcsec) would mostly affect the ratio in the clump edge where the emission is fainter. Alternatively, it is possible that the flux density at $250 \mathrm{GHz}$ has been systematically underestimated in the less intense emission areas. While reducing these data (following Massi et al. 2007), we noted some small changes in the source morphology depending on the choice of the source masking area. In turn, this suggests that low-level mm emission has not been fully recovered in our $250 \mathrm{GHz}$ map. A similar effect was noted by Massi et al. (2007) when comparing photometry of sources from their large-scale map of Vela D and from much smaller maps provided by other authors.

We can conclude that even in the HII region RCW 36, where regions with intense free-free emission are expected, the flux density ratio clearly indicates that the submm emission detected with LABOCA is dominated by dust thermal radiation with $\beta \sim 1-2$. External heating is also plausible, although our results are likely to be biased by systematic errors.

\subsection{Statistical properties of the submm cores}

In this section we discuss a few general properties of the submm cores by analysing the results obtained with both CuTEx and CLUMPFIND. The gas masses were derived for the starless and protostellar subsamples from Eq. (8) of Elia \& Pezzuto (2016) with a dust opacity $k_{v}(v=345 \mathrm{GHz})=0.86 \mathrm{~g}^{-1} \mathrm{~cm}^{2}$ (see Sect. 3.3). We adopted this value for immediate comparison of the derived masses with those obtained by Massi et al. (2007) in Vela D. To be consistent with the ATLASGAL catalogue (Schuller et al. 2009), our masses have to be scaled by a factor of 0.46. The single-temperature approximation of Sect. 4.4 is used for an easy comparison.

Following Massi et al. (2007), we examined the relationship between mass and size in the two samples, which is shown in Fig. 7. The maximum and minimum source sizes have been deconvolved and their geometrical average is displayed ( $x$ axis). Only sources with deconvolved apparent mean diameter larger than half the beam size have been plotted. From a linear fit in the $\log -\log$ space, we found $M \sim D^{1.7 \pm 0.1}$ for CLUMPFIND sources and a slightly steeper relation $\left(M \sim D^{2.2 \pm 0.1}\right)$ for CuTEx sources. The various biases affecting the results of different clump finding algorithms have long been known (e.g. Schneider \& Brooks 2004; Curtis \& Richer 2010); in this respect, CLUMPFIND

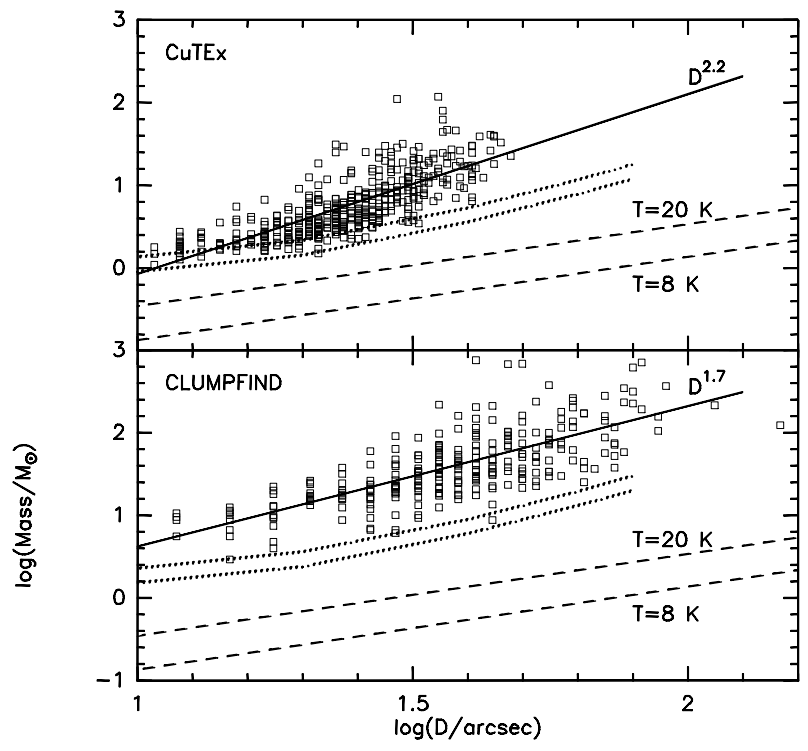

Fig. 7. Mass vs. size for the submm sources in Vela C. The mass is computed by assuming a constant $T_{\text {dust }}=12.5 \mathrm{~K}$. Top: cores retrieved with CuTEx, whereas bottom: the ones found with CLUMPFIND. The solid line is a linear fit (in the $\log -\log$ space) to the datapoints. The dotted lines are the detection limits estimated assuming rms values of 20 and $30 \mathrm{mJy} \mathrm{beam}^{-1}$ (see text). Finally, the dashed lines are the theoretical loci of Bonnor-Ebert critical half-masses at $T=8 \mathrm{~K}$ and $T=20 \mathrm{~K}$.

and CuTEx yield similar mass-size relations. In addition, the CuTEx datapoints spread over the same plot region as that of the Herschel data of Giannini et al. (2012; see their Fig. 5), although the latter do not exhibit a clear trend of increasing mass with size. As the detection limit depends on the apparent source size, detection loci can be derived as follows. By taking into account typical values of rms of 20 and $30 \mathrm{mJy} \mathrm{beam}^{-1}$ (see Sect. 3.1) and the pre-selection limit of three times the rms used for the CuTEx output, in the same assumptions as above, 0.06 and 0.09 Jy correspond to detection limits of $0.7 M_{\odot}$ and $1.1 M_{\odot}$, respectively, for point sources with $T_{\text {dust }}=12.5 \mathrm{~K}$. For extended sources with a 2D Gaussian spatial distribution of size $D$, this limit has to be scaled by $\left(B^{2}+D^{2}\right) / B^{2}$, where $B$ is the beam size. As for CLUMPFIND, the detection loci have been computed assuming 


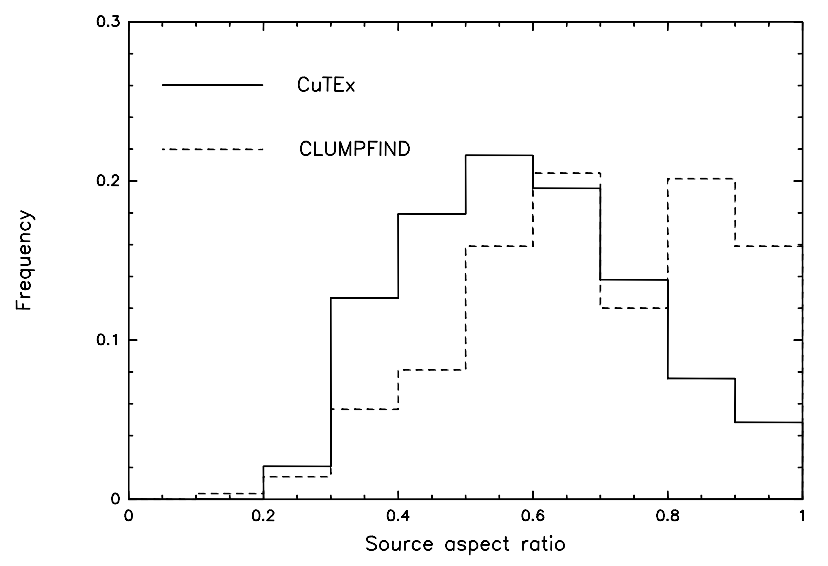

Fig. 8. Normalised distribution of ratios of minimum to maximum source size for the cores found by CuTEx (solid line) and the ones found by CLUMPFIND (dashed line). Only sources with minimum deconvolved size larger than half the LABOCA beam have been taken into account.

a detection limit of $5 \mathrm{rms}$, more consistent with the parameters used. These loci are highlighted in Fig. 7 by dotted lines. The more massive cores are clearly the bigger ones, although the lower envelope is affected by incompleteness. Therefore, the derived slopes may be in error and the mass-size relations might be slightly steeper.

Following Giannini et al. (2012), Fig. 7 also displays the theoretical loci of Bonnor-Ebert half critical masses $\left(M_{\mathrm{BE}}\right)$ for $T=8 \mathrm{~K}$ and $T=20 \mathrm{~K}$. Most of the cores clearly lie in the plot region where gravity dominates over thermal support. Although turbulence and magnetic field will provide further support, they are only likely to delay the collapse of cores that have not yet formed a protostar. This does not necessarily apply to cores hosting protostars, in which increased turbulence from outflows may eventually disrupt their structure.

In view of the filamentary structure of the cloud, it is of interest to have a look at the distribution of ratios of minimumto-maximum deconvolved source size, shown in Fig. 8. The peak between 0.5 and 0.6 suggests that the smallest structures tend to be prolate rather than filamentary. As shown in Fig. 8, the distribution of deconvolved source size ratios of the CLUMPFIND cores appears even less filamentary than that of the CuTEx cores. By plotting the distribution of the position angles of the CuTEx sources in the south, centre, and north parts of the cloud, we were unable to find any significant preferred orientation of the submm cores, which would also have pointed to a filamentary nature.

The total mass in dense cores retrieved by CuTEx is $\sim 5700 M_{\odot}$, whereas the total core mass retrieved by CLUMPFIND is $\sim 10000 M_{\odot}$. This is not unexpected because unlike CLUMPFIND, CuTEx is optimised for searching compact sources. Further, CLUMPFIND is able to include more low-level emission in a clump (see Fig. 7; also Cheng et al. 2018). Taking into account a total mass of $5.3 \times 10^{4} M_{\odot}$ from ${ }^{13} \mathrm{CO}$ emitting gas for Vela C (Yamaguchi et al. 1999), this indicates that $10-20 \%$ of the gas is in dense cores. This figure is consistent with the low star-formation efficiency of GMCs (Padoan et al. 2014) and the typical dense gas fraction in the inner Galaxy (e.g. Csengeri et al. 2016).

The mass distributions, or CMFs, are plotted in Fig. 9 for the CuTEx and CLUMPFIND samples. For both of them the CMF increases with decreasing mass down to a peak roughly located at the completeness limit of $M \sim 3.7 M_{\odot}$ (dotted line in figure), as estimated in Sect. 3.3. A linear $\chi^{2}$ fit (in the $\log -\log$ space) to this

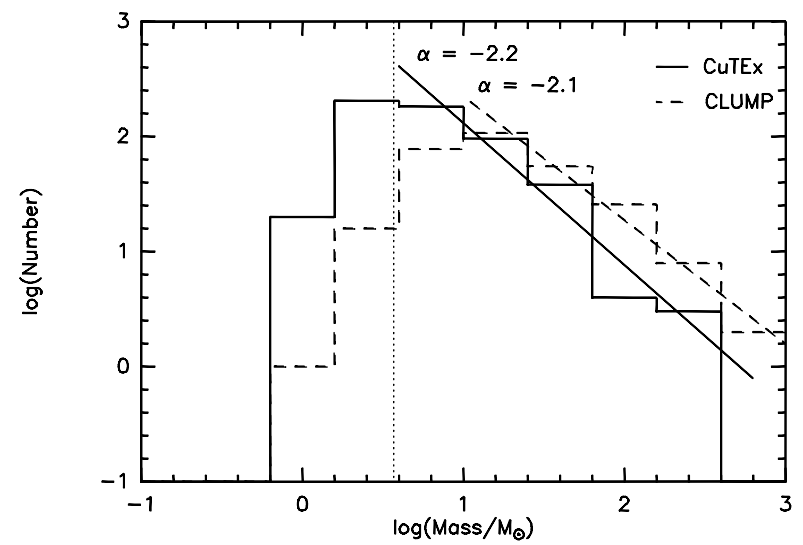

Fig. 9. Core mass function for the CuTEx sources (solid line) and the CLUMPFIND sources (dashed line). Also shown are linear fits to the CMFs corresponding to a relation $\mathrm{d} N / \mathrm{d} M \sim M^{-2.2}$ (CuTEx CMF; solid line) and $\mathrm{d} N / \mathrm{d} M \sim M^{-2.1}$ (CLUMPFIND CMF; dashed line), respectively. The vertical dotted line marks our estimated completeness limit.

part of the CMF yields a slope of $\alpha^{\prime}=-1.2 \pm 0.2$ (CuTEx) and $\alpha^{\prime}=-1.1 \pm 0.2$ (CLUMPFIND), where $\alpha^{\prime}=\alpha-1$ and $\alpha=2.35$ for a Salpeter IMF (Salpeter 1955; see Table 3 for definitions). We note that the histogram peaks have not been included in the fitted data range to minimise incompleteness effects.

As can also be deduced from Fig. 7, it appears that CuTEx tends to further subdivide the largest CLUMPFIND sources in smaller cores. Nevertheless, the power-law indices $\alpha$ are roughly consistent with each other at a $1 \sigma$ level. In the following we only discuss the results from the CuTEx algorithm without explicit mention. In addition, the submm cores located inside the sky area shown in Fig. 2 (hence likely to be associated with RCW 34) will not be taken into account.

\subsection{Starless and protostellar cores}

A further step in our analysis consists in separating the CuTEx sources into starless and protostellar cores and comparing the properties of the two subsamples. In principle, after gravitationally bound starless cores have evolved into protostellar cores they will lose part of their mass due to accretion onto the central object(s) and outflowing activity, developing temperature gradients as well. The two populations are therefore expected to exhibit different physical properties. In addition, if star formation proceeds in bursts, less evolved regions should display a larger fraction of starless cores, in contrast to more evolved regions. On the other hand, if star formation is continuous, the number of starless and protostellar cores will be related to the lifetimes in each stage.

Based on their association with WISE (and MSX) point sources, as explained in Sect. 3.4, we classified the submm sources into starless cores (328) and protostellar cores (221); a further refinement is explained in the following section. We note that cross-matching of LABOCA and WISE yielded a larger fraction of protostellar cores than Giannini et al. (2012) found in their Herschel sample, that is, $40 \%$ of 549 submm cores versus $18 \%$ of 268 Herschel cores.

Deriving the completeness limits of the WISE photometry is mandatory to assess the reliability of our catalogue of starless cores. We examined the histograms of the number of midinfrared (MIR) sources versus magnitude; taking into account the effects of the cuts required to fulfil the criteria of Koenig et al. (2012), rough completeness limits are [3.6] 14, [4.6] 12, 
[12] $\sim 9$ and [22] 7. These values are 1-3 mag brighter than the sensitivity limits quoted in the WISE Explanatory Supplement $^{3}$ for the relevant sky region. Once converted into flux units and, for example, compared with the models of Class I and Class 0 sources of $0.5 M_{\odot}$ by Whitney et al. (2004), it can be seen that the completeness limits at 3.6 and $4.6 \mu \mathrm{m}$ are faint enough to detect such objects taking into account a distance of $700 \mathrm{pc}$ and a further foreground reddening up to $A_{V}=20$. Even in the worst case of edge-on discs, these objects would be detectable at 3.6 and $22 \mu \mathrm{m}$. Furthermore, the completeness limit at $22 \mu \mathrm{m}$ is faint enough to allow detection of Class I and Class 0 sources of evenlower-mass central objects. Alternatively, one can compute the bolometric luminosity following Kryukova et al. (2012). Starting from our completeness limit at $22 \mu \mathrm{m}$, after conservatively dereddening it by $A_{V}=20$, we assumed a spectral index $\gamma=-2$ (see Table 3 for definition) to compute the MIR luminosity from Eq. (6) of Kryukova et al. (2012). Equation (7) of Kryukova et al. (2012) then yields $L_{\mathrm{bol}} \sim 1.7-2.8 L_{\odot}$, depending on whether the NIR flux is neglected (which may be the case) or extrapolated from $\gamma=-2$. A comparison with the birthline of Palla \& Stahler (1993) indicates a mass of $\sim 0.4-0.5 M_{\odot}$ for the central protostar. For the sake of comparison, we can roughly estimate the completeness limit in central masses of the Herschel protostellar cores in Giannini et al. (2012) using their quoted completeness limit at $70 \mu \mathrm{m}$ of $0.21 \mathrm{Jy}$ and following Dunham et al. (2008). By using Eq. (2) of Dunham et al. (2008), scaled to a distance of $700 \mathrm{pc}$, we found that the flux density at $70 \mu \mathrm{m}$ translates into a bolometric luminosity of the central (proto)star $L_{\text {bol }} \sim 0.28 L_{\odot}$ (we note that Dunham et al. 2008 indicate this luminosity as $\left.L_{\text {int }}\right)$. We highlight the fact that the $70 \mu \mathrm{m}$ emission is in principle a more sensitive protostellar tracer than WISE. However, this contrasts with the much lower number of protostellar cores found by Giannini et al. (2012), which may be due to a poorer effective sensitivity because of their selection criteria.

It is important to note that in principle the sample of starless cores will contain both transient unbound gas structures and real prestellar gas condensations. By considering the limited range of dust temperatures envisaged for the starless cores (see Sect. 4.4), a comparison between the location of the submm datapoints and the theoretical loci of Bonnor-Ebert critical half-masses $T=8$ and $20 \mathrm{~K}$ in Fig. 7 suggests that most of the submm starless cores are likely to be gravitationally bound. Therefore, although we refer to them as "starless", they are actually likely to be prestellar in nature.

\subsection{Core temperatures}

Given that the submm sources are in different evolutionary stages, and therefore have different temperatures, one should use the correct temperature for each object for an accurate mass determination. Based on Giannini et al. (2012), a few general trends are well established. These latter authors found a very narrow interval of dust temperatures, with means of $\sim 10$ and $\sim 13 \mathrm{~K}$ for their starless and protostellar cores, respectively. In particular, the temperature distribution for starless cores is strongly peaked at $\sim 10 \mathrm{~K}$, with a cut-off at $\sim 15 \mathrm{~K}$. Conversely, the temperature distribution of protostellar cores appears almost uniform between 9 and $15 \mathrm{~K}$, with a small number of warmer sources (up to $\sim 25 \mathrm{~K}$ ). Netterfield et al. (2009) analysed a much larger sample of sources using BLAST data $(250,350$, and $500 \mu \mathrm{m})$ from the whole $\left(\sim 50 \mathrm{deg}^{2}\right)$ Vela region. They found a temperature distribution ranging between 10 and $15 \mathrm{~K}$ for cores without

\footnotetext{
3 http://wise2.ipac.caltech.edu/docs/release/allsky/ expsup/
}

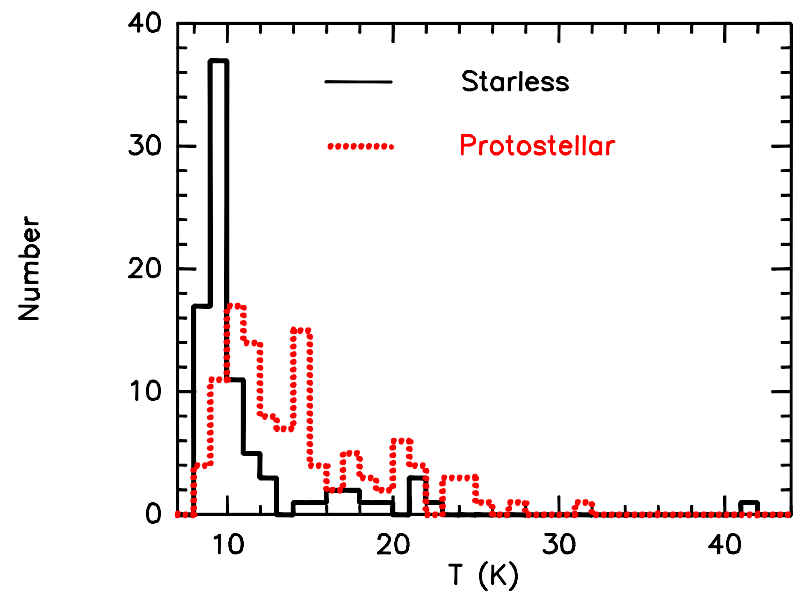

Fig. 10. Histograms of the dust temperatures of starless (solid black line) and protostellar (dotted red line) submm cores as derived from their FIR Herschel counterparts.

associated IRAS or MSX sources, similar to that of the starless sample of Giannini et al. (2012). However, the temperature distribution for all BLAST cores peaks at $\sim 12.5 \mathrm{~K}$ with $19 \%$ of the sources warmer than $20 \mathrm{~K}$. This is also confirmed by the temperature distribution derived by Hill et al. (2011) from the Herschel observations of Vela C. These latter authors found a bimodal temperature distribution, with a peak at $\sim 15 \mathrm{~K}$ and a tail extending to $\sim 30 \mathrm{~K}$. Remarkably, the southernmost regions are characterised by a strongly peaked distribution (with peak temperatures $<15 \mathrm{~K}$ ), and only the Centre-Ridge and the northernmost part exhibit a small fraction of warmer regions. Fissel et al. (2016) found (from the BLAST data) a number of lines of sight where dust appears heated by the HII region RCW 36 in the central part of Vela $\mathrm{C}$.

We take two different approaches to assign a dust temperature to each core. A first-order approximation widely used in the literature consists in assuming a single temperature for all sources. Given the small range of temperatures involved as discussed above, particularly for starless cores, a single value should represent a reasonable assumption. We chose $T_{\text {dust }}=12.5 \mathrm{~K}$ for this first-order approximation. This value would underestimate the mass of cores with $T_{\text {dust }}=10 \mathrm{~K}$ by only $35 \%$, and overestimate the mass of cores with $T_{\text {dust }}=15 \mathrm{~K}$ by only $37 \%$. Undoubtedly, a number of warm sources $\left(T_{\text {dust }}>15-20 \mathrm{~K}\right)$ are expected in our sample, but many of them are probably located in areas near stellar sources radiating intense UV fields, such as the HII region RCW 36, and represent only a small percentage of the total.

The second approach consists in deriving single-core dust temperatures for the 197 Herschel compact sources associated with submm cores as described in Sect. 3.5. For each Herschel source we performed flux scaling at the various Herschel wavelengths (a method adopted by Motte et al. 2010) and then fitted a greybody to the SED, both explained in Giannini et al. (2012). The submm points are not included in the fit. We also note that the Herschel HPBW is larger than the one of LABOCA for $\lambda \geq 350 \mu \mathrm{m}\left(\sim 25^{\prime \prime}\right.$ at $350 \mu \mathrm{m}$ and $\sim 36^{\prime \prime}$ at $\left.500 \mu \mathrm{m}\right)$. In principle, unresolved cold dust structures might bias the derived temperature, but this should be mitigated by the flux scaling in most cases. At the end of the process, we were able to derive a dust temperature for 86 of the 328 submm starless cores and 111 of the 221 submm protostellar cores of our sample.

Figure 10 shows single-core temperature histograms for the submm starless and protostellar cores. The two distributions are 


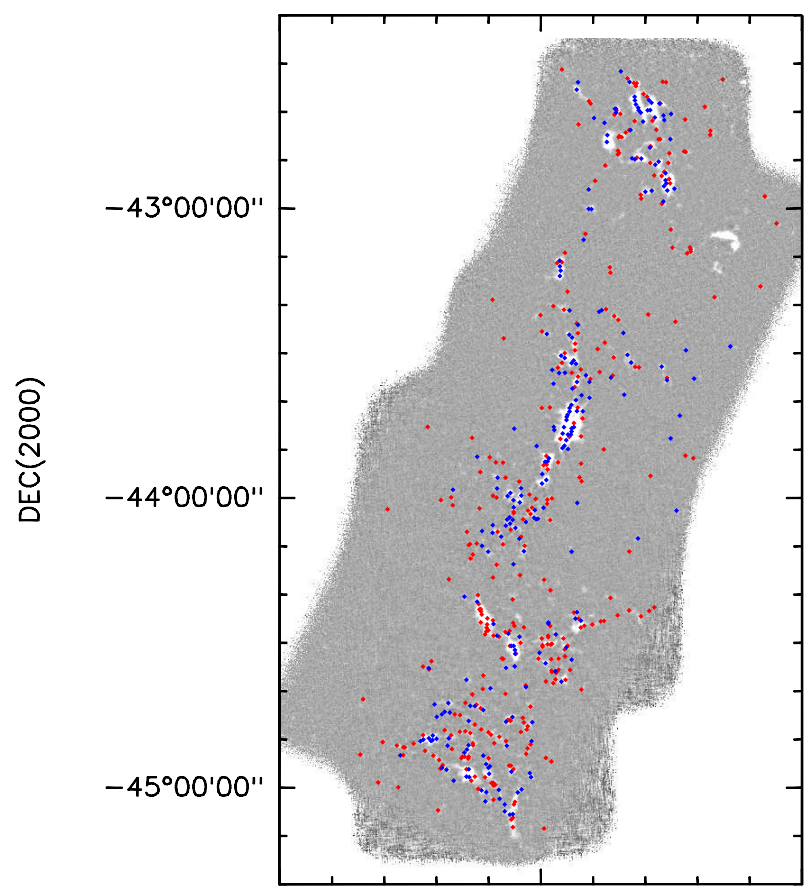

$\operatorname{RA}(2000)$

Fig. 11. Projected spatial distribution of starless cores (red diamonds) and protostellar cores (blue diamonds) overlaid on the LABOCA map of $345 \mathrm{GHz}$ emission from Vela $\mathrm{C}$.

clearly different, with starless cores exhibiting a strong peak at $\sim 9 \mathrm{~K}$ and protostellar ones peaking at $\sim 11 \mathrm{~K}$ (the other peak at $\sim 15 \mathrm{~K}$ disappears after shifting the bin centres by $0.5 \mathrm{~K}$ ) and exhibiting a significant tail at higher temperatures. A look at the 12 starless cores with a temperature $>14 \mathrm{~K}$ indicates that all but one have been detected at $70 \mu \mathrm{m}$ and suggests that these are probably protostellar sources contaminating the starless sample. This further constrains the temperature of the starless cores in the range 9-12 $\mathrm{K}$ and enhances the difference between the two distributions, confirming that the two samples are different in nature. A comparison with Fig. 4 of Giannini et al. (2012) shows that our temperature distributions are not very different from theirs, although their statistics are probably biased by their much smaller sample of protostellar objects.

We note that the limited number of submm sources associated with a Herschel source prevents us from using the whole sample of submm cores. However, if the temperature distribution of starless cores is representative of all starless cores in the region, using the right temperature for each source is not strictly necessary. In this respect, the single-temperature approach allows us to use the whole submm sample to explore its statistical properties.

In the end, the 12 starless sources with $T>14 \mathrm{~K}$ were re-classified as protostellar. Therefore, the final subsamples are composed of 316 starless and 233 protostellar cores. The spatial distribution of starless and protostellar cores is shown in Fig. 11. We use the same symbols as Giannini et al. (2012) for comparison with their Fig. 1. It is also interesting to derive the percentage of protostellar cores in different sky areas. This confirms the visual impression that the southern part of Vela $\mathrm{C}$ hosts the lowest fraction of protostellar cores $(\sim 35 \%$ at Dec $\left.<-44^{\circ} 15^{\prime}\right)$, while the central part hosts the largest fraction $\left(52 \%\right.$ at $\left.-44^{\circ} 15^{\prime}<\operatorname{Dec}<-43^{\circ} 10^{\prime}\right)$. At Dec $>-43^{\circ} 10^{\prime}, \sim 41 \%$ of the submm cores are protostellar.

\subsection{Core masses: individual and statistical properties}

The masses of the submm cores were derived from their $345 \mathrm{GHz}$ flux density by using Eq. (8) of Elia \& Pezzuto (2016), with a dust opacity $k_{v}=0.86 \mathrm{~g}^{-1} \mathrm{~cm}^{2}$ (see Sect. 3.3). Dust temperatures were assigned based on the two approaches outlined in Sect. 4.4, and we assumed a gas-to-dust ratio of 100.

Errors on the mass estimates are due to uncertainties on distance, dust opacity, temperature, and flux calibration. The effects of distance errors are tabulated in the appendix. Dust opacity is discussed by Ossenkopf \& Henning (1994) who conclude that uncertainties are unlikely to exceed a factor of approximately two, even when different physical environments are taken into account. Having selected the same molecular cloud and specific classes of objects (namely prestellar and protostellar cores), this should not be the case within each of the classes. These authors show that, providing cores remain stable over a timescale of $\sim 10^{5} \mathrm{yr}$, opacity gains only a little dependence on gas density due to dust coagulation. The mass-size relation that we find for all cores implies that their mean gas densities range within an order of magnitude, that is, $\sim 10^{5}-10^{6} \mathrm{~cm}^{-3}$. It can be seen from Ossenkopf \& Henning (1994; their Tables 2 and 3) that opacity variations are less than $30 \%$ for densities in the range $10^{5}-10^{6} \mathrm{~cm}^{-3}$ (less than $20 \%$ for densities of $10^{6}-10^{8} \mathrm{~cm}^{-3}$ ) and dust grains with ice mantles. One might suppose that the main error contributed by dust opacity would result in a zero-order term which would translate into a mass scale factor (probably $\approx 2$ ), as for distance errors. This can be caused by an incorrect choice of the most suitable dust models for the class of objects considered. We also note that the scaling factors to use if simultaneously adopting the ATLASGAL dust opacity and the revised distance of $950 \mathrm{pc}$ would partly cancel each other. Flux calibration errors are more likely to affect all sources in a relatively large cloud area in the same way, rather than yield random source-tosource flux fluctuations. As stated in Sect. 3.1, we found that the fluxes of the submm sources are consistent within $30 \%$ when observed in different runs. Having mapped most of Vela $\mathrm{C}$ a few times, the actual calibration accuracy should be better than that. However, even a mass variation of $30 \%$ due to calibration or dust emissivity errors turns into a variation of the logarithm of mass of $\sim 0.1$, which has to be compared, for example, with the logarithmic size of the bins we used in the mass histograms: 0.4 . In conclusion, although core masses may even be systematically incorrect by up to a factor of approximately two, parameters such as the CMF slope are unaffected by distance uncertainties and are only very mildly affected by dust opacity or calibration uncertainties; this is especially true for prestellar cores which exhibit only small temperature differences and are not expected to have inner temperature gradients (making their physical environment relatively constant). The derived masses also depend on the adopted dust temperature and, as seen above, errors of a few Kelvin cause significant changes. The effects of temperature on the $\mathrm{CMF}$ are therefore discussed in more detail later.

Table 4 compares the statistical properties of the various samples of sources, namely the Herschel FIR sources from Giannini et al. (2012), the LABOCA submm sources with temperature derived from matching them to Herschel sources, and the LABOCA submm sources with an assumed constant temperature. Each submm subsample (starless and protostellar) clearly exhibits similar properties irrespective of the assumed temperature. The most notable difference lies in the high-mass end of the mass distributions. We checked that only 7 submm protostellar sources have a mass $>50 M_{\odot}$ when computed assuming $T_{\text {dust }}=12.5 \mathrm{~K}$. Six of them are included in the sample of submm 
Table 4. Statistical properties of submm and FIR (Herschel) sources in Vela C.

\begin{tabular}{|c|c|c|c|c|c|c|c|c|c|}
\hline \multirow[t]{2}{*}{ Parameter } & \multicolumn{3}{|c|}{$\begin{array}{c}\text { Herschel } \\
\text { Starless (218) }\end{array}$} & \multicolumn{3}{|c|}{$\begin{array}{c}\text { LABOCA+ Herschel } \\
\text { Starless }(73)\end{array}$} & \multicolumn{3}{|c|}{$\begin{array}{c}\text { LABOCA constant } T_{\text {dust }} \\
\text { Starless (316) }\end{array}$} \\
\hline & Median & Average & Min-max & Median & Average & $\operatorname{Min}-\max$ & Median & Average & Min-max \\
\hline$M\left(M_{\odot}\right)$ & 3.3 & 5.5 & $0.13-55.8$ & 6.6 & 9.9 & $1.5-43.6$ & 3.9 & 6.1 & $0.8-127.9$ \\
\hline$T_{\text {dust }}(\mathrm{K})$ & 10.0 & 10.3 & $8.0-15.2$ & 9.5 & 9.6 & $8.0-12.7$ & 12.5 & - & - \\
\hline \multirow[t]{3}{*}{$D(\mathrm{pc})$} & 0.064 & 0.067 & $0.025-0.13$ & 0.080 & 0.084 & $\left(<D_{\text {beam }}\right)-0.151$ & 0.078 & 0.082 & $\left(<D_{\text {beam }}\right)-0.151$ \\
\hline & \multicolumn{3}{|c|}{ Protostellar (48) } & \multicolumn{3}{|c|}{ Protostellar (124) } & \multicolumn{3}{|c|}{ Protostellar (233) } \\
\hline & Median & Average & Min-max & Median & Average & Min-max & Median & Average & Min-max \\
\hline$M\left(M_{\odot}\right)$ & 2.7 & 4.8 & $0.15-29.1$ & 8.2 & 14.4 & $0.6-124.2$ & 8.0 & 16.3 & $0.7-289.7$ \\
\hline$T_{\text {dust }}(K)$ & 11.4 & 12.8 & $9.0-24.2$ & 14.2 & 15.0 & $8.4-41.0$ & 12.5 & - & - \\
\hline$D(\mathrm{pc})$ & 0.040 & 0.040 & $0.025-0.07$ & 0.091 & 0.095 & $0.040-0.155$ & 0.093 & 0.094 & $0.032-0.162$ \\
\hline
\end{tabular}

Notes. Median, average, maximum, and minimum values are given for each physical parameter. Data for the Herschel sources are from Giannini et al. (2012).

Table 5. Spectral indices $\alpha$ (with $\mathrm{d} N / \mathrm{d} M \sim M^{\alpha}$ ) from linear fits and the ML estimator.

\begin{tabular}{|c|c|c|c|c|c|c|c|}
\hline \multirow[b]{2}{*}{$\mathrm{CMF}$} & \multirow[b]{2}{*}{$\begin{array}{c}\text { Temperature } \\
(\mathrm{K})\end{array}$} & \multicolumn{2}{|c|}{ Protostellar+starless } & \multicolumn{2}{|c|}{ Protostellar } & \multicolumn{2}{|c|}{ Starless } \\
\hline & & Linear fit & ML & Linear fit & ML & Linear fit & ML \\
\hline CuTEx & 12.5 & $-2.2 \pm 0.2$ & $-2.2 \pm 0.1$ & $-2.0 \pm 0.1$ & $-1.9 \pm 0.1$ & $-2.4 \pm 0.2$ & $-2.8 \pm 0.2$ \\
\hline CLUMPFIND & 12.5 & $-2.1 \pm 0.2$ & $-2.0 \pm 0.1$ & - & - & - & - \\
\hline CuTEx & Herschel match & - & - & $-2.2 \pm 0.2$ & $-1.9 \pm 0.1$ & $-2.1 \pm 0.4$ & $-2.0 \pm 0.2$ \\
\hline CuTEx & Monte Carlo & - & - & $-2.1 \pm 0.1$ & - & $-2.7 \pm 0.1$ & - \\
\hline CuTEx & $3 \mathrm{~T}^{(a)}$ & - & - & $-2.5 \pm 0.2$ & $-2.2 \pm 0.2$ & - & - \\
\hline CuTEx (north) & 12.5 & - & - & $-2.2 \pm 0.5$ & $-1.5 \pm 0.1$ & $-2.3 \pm 0.4$ & $-2.1 \pm 0.3$ \\
\hline CuTEx (centre) & 12.5 & - & - & $-1.9 \pm 0.2$ & $-1.7 \pm 0.1$ & $-2.3 \pm 0.2$ & $-2.2 \pm 0.3$ \\
\hline CuTEx (south) & 12.5 & - & - & $-2.6 \pm 0.3$ & $-2.5 \pm 0.2$ & $-2.3 \pm 0.2$ & $-2.7 \pm 0.3$ \\
\hline
\end{tabular}

Notes. To minimise incompleteness effects, the adopted lower-mass ends are $\log \left(M / M_{\odot}\right)=0.8-1.2$ for the fits (i.e. the central value of the bin next to the peak) and $\log \left(M / M_{\odot}\right)=0.8$ for the ML computations (1.2 and 1.2, respectively, for CLUMPFIND). ${ }^{(a)}$ Three different dust temperatures were used: $12.5 \mathrm{~K}$ for cores in the south, $20 \mathrm{~K}$ for cores in the centre, and $16 \mathrm{~K}$ for cores in the north of Vela $\mathrm{C}$ (see text).

protostellar sources with temperature from Herschel data, but having been assigned a higher $T_{\text {dust }}$ they exhibit lower masses here. On the other hand, only two submm starless sources have a mass $>40 M_{\odot}$ when computed assuming $T_{\text {dust }}=12.5 \mathrm{~K}$. Neither of them is included in the sample of submm starless sources with a temperature from Herschel data. Both are located in the area of RCW 36, a region with a high surface density of submm sources.

By comparing the median mass of the two submm starless subsamples (i.e. submm plus Herschel and submm with constant temperature), we note that this roughly decreases to our estimated completeness limit when we use the whole starless sample assuming a constant $T_{\text {dust }}$. This indicates that many submm starless sources without an FIR-derived temperature lie below the completeness limit.

In addition, we performed a series of comparisons between different instances of CMFs, all of which assume that they are linear in the log-log space above the mass completeness limit. In this respect, we exploit two different ways of computing the CMF slope $\alpha$ : by a simple linear fit on a histogram, and by using an unbiased statistical estimator, namely the maximum likelihood (ML) estimator (Maschberger \& Kroupa 2009). The former makes for an easier interpretation, but the latter is less prone to statistical bias. The results are listed in Table 5. The CMFs obtained for the submm starless and protostellar cores with dust temperature derived from Herschel data are plotted in

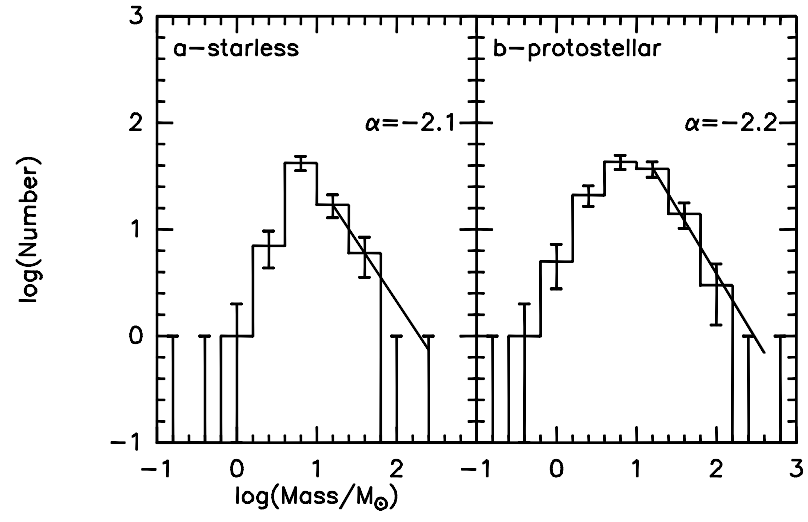

Fig. 12. Core mass function for the submm starless sources (left) and the submm protostellar sources (right) whose temperature was derived from Herschel data. Also shown, linear fits to the CMFs corresponding to a relation $\mathrm{d} N / \mathrm{d} M \sim M^{-2.1}$ (starless cores) and $\mathrm{d} N / \mathrm{d} M \sim M^{-2.2}$ (protostellar cores), respectively.

Fig. 12. The linear fit to the high-mass end yields $\alpha=-2.1 \pm 0.4$ for the starless sample and $\alpha=-2.2 \pm 0.2$ for the protostellar sample. On the other hand, the CMF of starless and protostellar cores derived for a constant temperature of $12.5 \mathrm{~K}$ are 


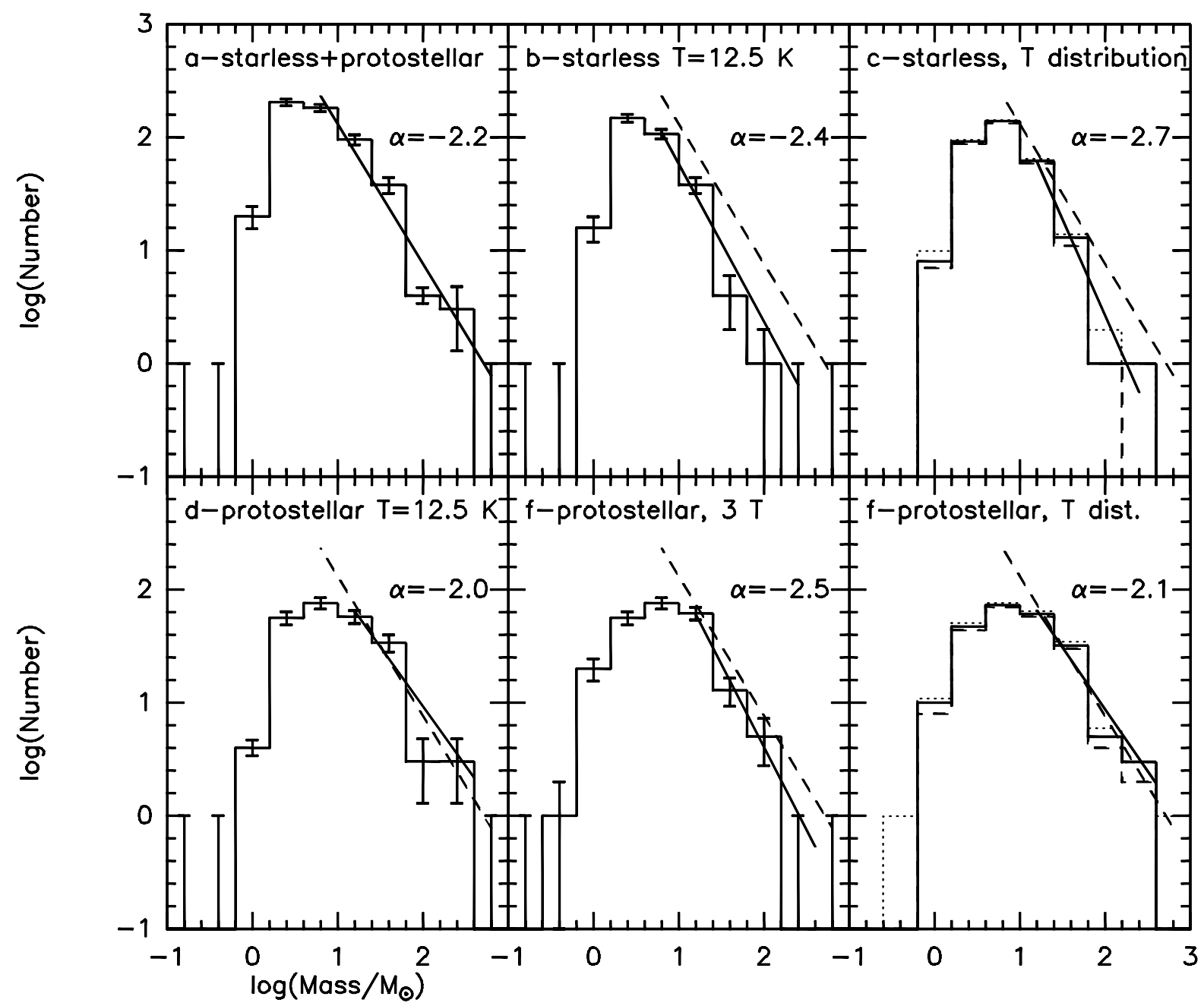

Fig. 13. Comparison between core mass functions from the CuTEx starless and protostellar source samples based on different choices of dust temperatures. Solid straight lines are linear fits to the high-mass ends, and dashed straight lines show the slope found from the same fit to the total sample (starless plus protostellar). $a$ : same as in Fig. 9; $b$ : starless cores with a constant dust temperature of $12.5 \mathrm{~K}$; $c$ : starless cores with temperature assigned based on a Monte Carlo procedure (see text); $d$ : protostellar cores with a constant dust temperature of $12.5 \mathrm{~K}$; $e$ : protostellar cores with three different temperatures assigned based on the core location (see text); $f$ : protostellar cores with temperature assigned based on a Monte Carlo procedure (see text). In panels $c$ and $f$, the solid line histogram is the median (second quartile) distribution, the long-dashed line histogram is the first quartile distribution and the short-dashed line the third quartile distribution. In the other panels, errorbars indicate the Poissonian standard deviations of counts in each bin. Also indicated in each panel is the $\alpha$ index resulting from the linear fits, where $\mathrm{d} N / \mathrm{d} M \sim M^{\alpha}$.

shown in Figs. 13b and d. The linear fit yields $\alpha=-2.4 \pm 0.2$ (starless cores) and $\alpha=-2.0 \pm 0.1$ (protostellar cores). The most remarkable difference between assuming a constant temperature and using Herschel dust temperatures consists in the slightly steeper $\alpha$ of the starless constant-temperature sample, although both slopes are consistent within $1 \sigma$. However, the ML estimator results in an even steeper starless CMF ( $\alpha=-2.8 \pm 0.2)$, above a $2 \sigma$ significance level. By comparing Figs. 13b and 12a one can note that the single-core temperature approach (i.e. matching of Herschel and LABOCA sources) misses a fraction of objects around the peak of the single-temperature CMF.

To further test the effects of temperature, we used the socalled bootstrap method adopting the temperature distributions of starless and protostellar Herschel cores obtained by Giannini et al. (2012). Thus, we developed a simple Monte Carlo procedure to assign a temperature drawn from these latter distributions to each source of our starless and protostellar samples. We constructed 1000 realisations each of the two CMFs. The first three quartiles of each bin are shown in Figs. 13c and f. A linear fit to the higher-mass end of the median distributions (second quartile, which conserves the total number of sources in both cases) yields $\alpha=-2.7 \pm 0.1$ (starless cores) and $\alpha=-2.1 \pm 0.1$ (protostellar cores), consistent with those derived from Figs. $13 \mathrm{~b}$ and d. In particular, this procedure enhances the higher steepness of the starless CMF. The distributions corresponding to the first and third quartiles show that the error per bin expected from temperature core-to-core variations is similar to that due to the Poissonian count statistics.

We conclude that as long as the dust temperatures are randomly distributed among the cores, this does not add further uncertainty to the CMF compared to that expected from the Poissonian statistics. A similar result was obtained by Csengeri et al. (2017) on a smaller sample of clumps using Monte Carlo methods. However, systematic differences in temperatures could still significantly affect the CMFs. Based on the temperature distributions shown in Fig. 10, systematic temperature differences may be expected to significantly affect only the protostellar $\mathrm{CMF}$, since the spread in temperature is relatively narrow for 


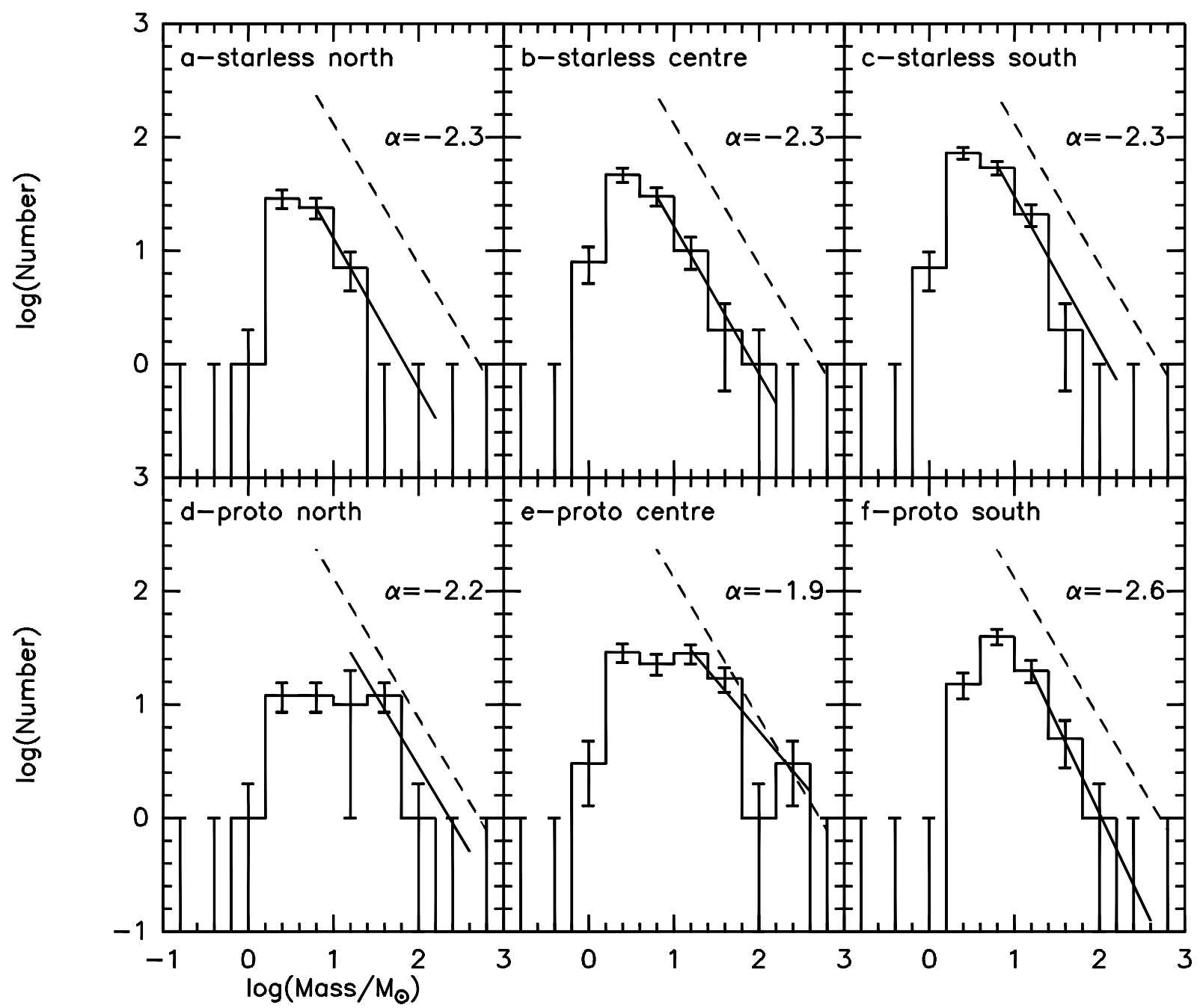

Fig. 14. Comparison between core mass functions from the CuTEx starless and protostellar samples obtained in different parts of the cloud with a constant temperature of $12.5 \mathrm{~K}$. Solid straight lines are linear fits to the high-mass ends, dashed straight lines show the slope found from the same fit to the total sample (starless plus protostellar; see Fig. 13a). $a$ : only starless sources in the northern part (Dec $\left.>-43^{\circ} 10^{\prime}\right) ; b$ : only starless sources in the central part $\left(-44^{\circ} 15^{\prime}<\right.$ Dec $\left.<-43^{\circ} 10^{\prime}\right) ; c$ : only starless sources in the southern part $\left(\operatorname{Dec}<-44^{\circ} 15^{\prime}\right) ; d$ : only protostellar sources in the northern part $\left(\operatorname{Dec}>-43^{\circ} 10^{\prime}\right)$; $e$ : only protostellar sources in the central part $\left(-44^{\circ} 15^{\prime}<\right.$ Dec $\left.<-43^{\circ} 10^{\prime}\right)$; $f$ : only protostellar sources in the southern part (Dec $<-44^{\circ} 15^{\prime}$ ). Error bars indicate the Poissonian standard deviations of counts in each bin. Also indicated in each panel is the $\alpha$ index resulting from the linear fits, where $\mathrm{d} N / \mathrm{d} M \sim M^{\alpha}$.

the starless subsample. The only systematic difference that can be envisaged is a temperature gradient from south to north (e.g. Fissel et al. 2016). To test the effects, we derived the CMF of protostellar cores by assigning a constant temperature of $12.5 \mathrm{~K}$ for Dec $<-44^{\circ}, 20 \mathrm{~K}$ for $-44^{\circ}<$ Dec $<-43^{\circ} 30^{\prime}$, and $16 \mathrm{~K}$ for Dec $>-43^{\circ} 30^{\prime}$. The results are shown in Fig. 13e. A linear fit to the high-mass end of the protostellar CMF yields $\alpha=-2.5 \pm 0.2$, steeper than all other determinations of the protostellar CMF (see Figs. 13d and $\mathrm{f}$ and Table 5). On the other hand, the ML estimator yields $\alpha=-2.2 \pm 0.2$, suggesting no significant differences.

We also wanted to investigate spatial changes in the CMF throughout Vela C. Therefore, we constructed the CMFs for a constant dust temperature of $12.5 \mathrm{~K}$ by selecting sources in the three areas defined in Sect. 4.4 (north, centre, and south). We note that the number of sources are comparable in the centre and south areas (104 starless and 95 protostellar cores in the centre, 152 starless and 81 protostellar cores in the south), but are roughly half as much in the north (69 starless and 48 protostellar cores). The results are shown in Fig. 14. As for the starless samples, their CMFs do not exhibit any significant variations above a $1 \sigma$ level with position. The slopes of the high-mass end of the protostellar CMFs are flatter in the north and centre than in the south. The ML estimator confirms this trend at a higher confidence level. We also note that any systematic difference in the temperature of protostellar cores between the three areas, as tested above, would only shift the CMFs in $\log$ (mass), but would not affect the CMF shapes and slopes.

One concern is that the protostellar and starless CMFs in the centre may be affected by the saturation in the WISE band and the use of MSX towards RCW 36. A look at the submm cores in this area shows that only four starless cores are above the mass completeness limits, three of which have masses (in the singletemperature approximation) of 128,44 , and $39 M_{\odot}$, respectively. The only effect that one can envisage is that some or all of these are actually protostellar in nature. This would at most result in a further steepening of the starless CMF and a further flattening of the protostellar CMF in the centre. We also assessed how the poorer statistics in the three areas may bias the results by performing new fits after shifting the (north, centre, south) CMF bins by 0.2 (in logarithm). We obtained CMFs that are slightly 


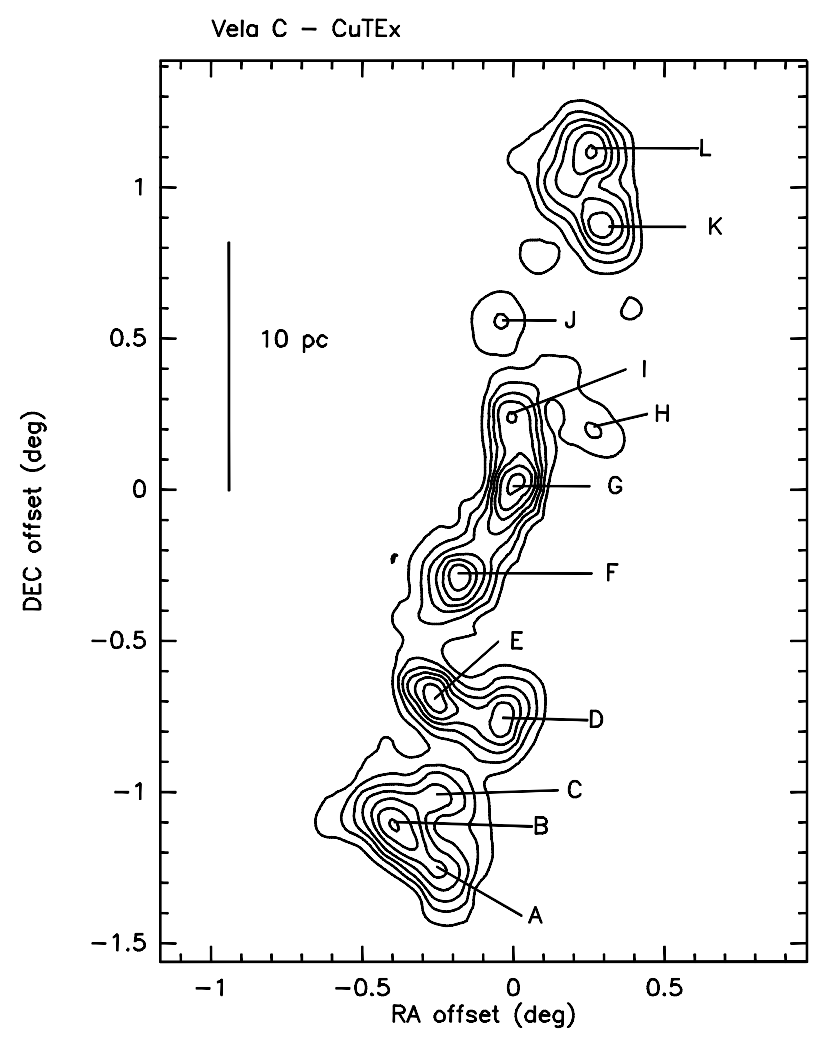

Fig. 15. Surface density map of CuTEx sources down to $0.3 \mathrm{Jy}$ of flux density. Contours range from 100 sources $\mathrm{deg}^{-2}$ in steps of 150 sources $\mathrm{deg}^{-2}$. Coordinate offsets are with respect to the position of the ionisation source of RCW $36\left(\mathrm{RA}=08^{\mathrm{h}} 59^{\mathrm{m}} 27^{\mathrm{s}}, \mathrm{Dec}=-43^{\circ} 45^{\prime} 26^{\prime \prime}\right)$.

flatter in all cases (the point at $\log \left(M / M_{\odot}\right)=0.6$ was included in the linear fits), but consistent with the previous determinations at a $1 \sigma$ level.

\subsection{Clustering and large-scale structures}

To probe core clustering in Vela $\mathrm{C}$, we counted all CuTEx sources down to a flux density of $0.3 \mathrm{Jy}$ (the estimated completeness limit) inside squares of side $\sim 9^{\prime}(\sim 1.8 \mathrm{pc}$ at $700 \mathrm{pc})$ parallel to the right ascension axis, offset by half the square side (i.e. a Nyquist sampling interval) in both right ascension and declination. The resulting map of surface density is shown in Fig. 15. Clearly, at least 12 clusters of cores can be retrieved; these clusters are almost equally spaced and have sizes of $\sim 2 \mathrm{pc}$ (therefore unresolved). This probably reflects large-scale fragmentation of the pristine molecular cloud. Positions and peak source surface densities of the clusters are listed in Table 6.

A look at Fig. 11 suggests that protostellar cores may concentrate towards the filaments. By repeating the same procedure as above for starless and protostellar cores separately (after decreasing the square size to 4.5) we could not find any significant morphological difference in the lowest density contours. Nevertheless, starless and protostellar projected distributions appear to peak at slightly different locations (see Fig. 16). This hints at least at a partial segregation of regions hosting starless and protostellar cores. Furthermore, a comparison of the submm and FIR maps suggests a population of starless, low-mass dust cores (below our submm completeness limit) outside the cloud filaments (see Sect. 4.4) that should be further investigated with more sensitive submm observations.
Table 6. Position (offsets from RA $=08^{\mathrm{h}} 59^{\mathrm{m}} 27^{\mathrm{s}}$, Dec $=-43^{\circ} 45^{\prime} 26^{\prime \prime}$ ) and peak surface density of the clusters of submm cores in Vela C.

\begin{tabular}{cccc}
\hline \hline $\begin{array}{c}\text { Cluster } \\
\text { ID }\end{array}$ & $\begin{array}{c}\text { RA offset } \\
(\mathrm{deg})\end{array}$ & $\begin{array}{c}\text { DEC offset } \\
(\mathrm{deg})\end{array}$ & $\begin{array}{c}\text { Peak surf. density } \\
\left(\mathrm{pc}^{-2}\right)\end{array}$ \\
\hline A & -0.25 & -1.26 & 5 \\
B & -0.40 & -1.11 & 7 \\
C & -0.25 & -1.02 & 4 \\
D & -0.03 & -0.76 & 5 \\
E & -0.26 & -0.69 & 6 \\
F & -0.18 & -0.29 & 7 \\
G & 0.01 & 0.03 & 7 \\
H & 0.27 & 0.20 & 2 \\
I & 0.00 & 0.24 & 5 \\
J & -0.04 & 0.56 & 2 \\
K & 0.29 & 0.88 & 5 \\
L & 0.26 & 1.12 & 6 \\
\hline
\end{tabular}

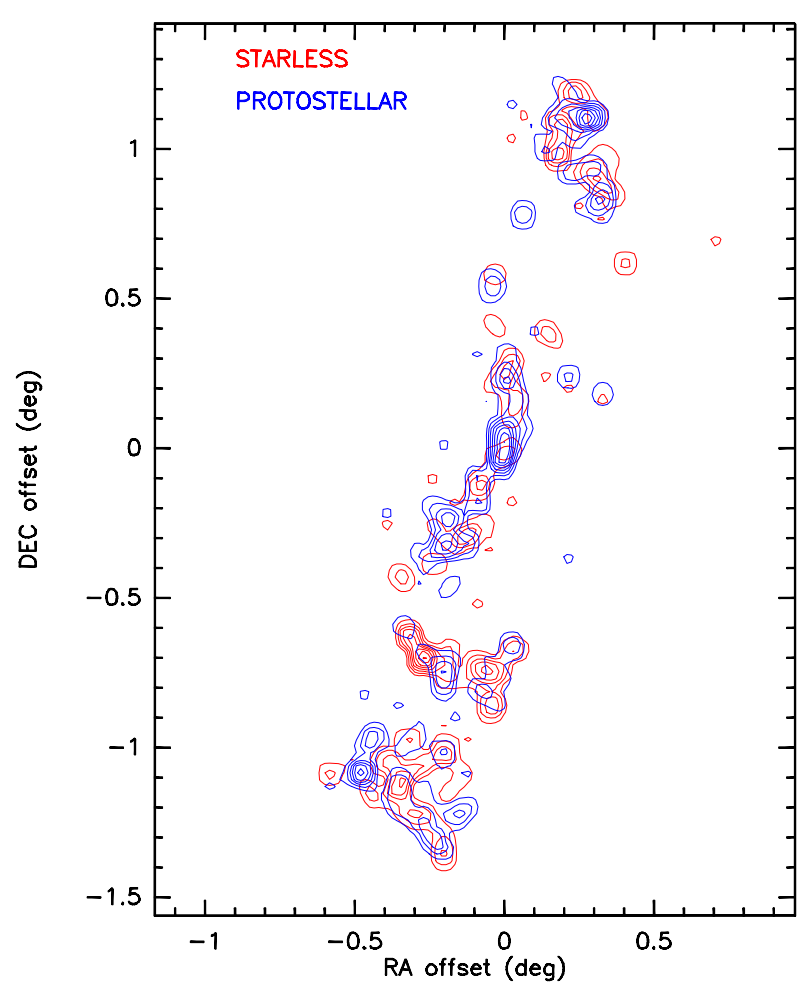

Fig. 16. As in Fig. 15, but for starless (red contours) and protostellar (blue contours) cores separately. Only the highest contours are plotted (from 200 sources deg ${ }^{-2}$ in steps of 200 sources $\mathrm{deg}^{-2}$ ). Coordinate offsets are with respect to the position of the ionisation source of RCW 36 $\left(\mathrm{RA}=08^{\mathrm{h}} 59^{\mathrm{m}} 27^{\mathrm{s}}\right.$, Dec $\left.=-43^{\circ} 45^{\prime} 26^{\prime \prime}\right)$.

\section{Discussion}

\subsection{Comparison with other studies of the VMR}

The submm CMFs can easily be compared with those derived for Vela C in other works. Netterfield et al. (2009) derived powerlaw indices $\alpha=-1.95 \pm 0.05$ for sources with $T_{\text {dust }}>14 \mathrm{~K}$ and $\alpha=-3.22 \pm 0.14$ for sources with $T_{\text {dust }}<14 \mathrm{~K}$ from their BLAST FIR observations of the whole Vela region. The first value must be compared with that derived from the linear fits to our protostellar sample and in fact is fully consistent with it (see Table 5). On the other hand, the second value is not consistent 
with our linear fits to the starless sample. Nevertheless, we note that Netterfield et al. (2009) derived $\alpha=-2.9 \pm 0.2$ for cold cores in Vela $\mathrm{C}$, which is closer to our linear fit $(\alpha=-2.4 \pm 0.2$, see Table 5) and quite consistent with the ML value $(\alpha=-2.8 \pm 0.2)$ for starless cores in single-temperature approximation.

Giannini et al. (2012) found $\alpha=-2.1 \pm 0.2$ for their sample of Herschel prestellar cores in Vela C, which is fully consistent with our linear fit to the subsample of starless cores with singlecore temperatures $(\alpha=-2.1 \pm 0.4$, see Table 5). These authors note that by limiting their fit to the CMF at $M>14 M_{\odot}$, the completeness limit of BLAST data, one obtains $\alpha=-2.9 \pm 0.2$, which is consistent with our ML value for the starless sample in single-temperature approximation. This would confirm that the Herschel sample is missing a significant fraction of low-mass $\left(M<14 M_{\odot}\right)$ cores compared to the submm sample, which would explain the discrepancy between the CMFs of starless cores in the single-temperature approximation and the single-core temperatures (i.e. from the associated Herschel cores) already noted ( $\alpha \sim-2.4,-2.8$ vs. $\alpha \sim-2.1)$; although this difference is only marginally significant.

As for other clouds in the VMR, Massi et al. (2007) found $\alpha=-1.45$ using CLUMPFIND on their mm map $(250 \mathrm{GHz})$ of Vela D. As they do not differentiate between starless and protostellar cores, this value must be compared to the value that we obtained from the whole sample using the same algorithm, that is, $\alpha=-2.1 \pm 0.2$ (see Table 5). They claim a mass completeness limit of $1-1.3 M_{\odot}$ and their most massive core is $\sim 100 M_{\odot}$, less than found in Vela $\mathrm{C}$ with CLUMPFIND. However, Olmi et al. (2009) revised the power-law index for Vela D to $\alpha=-2.3 \pm 0.2$ by combining the data of Massi et al. (2007) and BLAST FIR photometry. They concluded that the value derived by Massi et al. (2007) is likely to be biased due to incompleteness. The mass spectra of dense cores in the two clouds are therefore consistent with each other, as far as protostellar and starless cores are considered together.

We compared the mass of a few submm cores with those derived by Minier et al. (2013) for seven clumps identified in the same area (towards RCW 36). We found some discrepancies that are discussed in Appendix B and are probably due to the different measuring techniques and clump/core definition adopted.

Other aspects of our results can be explored through a comparison with the sample of IR cores of Giannini et al. (2012). The most remarkable difference between the Herschel FIR and LABOCA submm statistics (see Table 4) is that the sources in the protostellar sample are on average more massive than the starless sources, as far as submm cores are concerned. In addition, the FIR sources are on average smaller than the submm ones, which is clearly due to using the more resolved $160 \mu \mathrm{m}$ map to set the Herschel source size. The deconvolved sizes of both starless and submm protostellar sources are plotted in Fig. 17. This can be compared with Fig. 4 of Giannini et al. (2012). The distribution of the submm starless cores resembles that of the Herschel starless cores, peaking at $\sim 0.08 \mathrm{pc}$ (compared to $0.05 \mathrm{pc}$ ) and declining at $D>0.1 \mathrm{pc}$. On the other hand, protostellar submm cores display a size distribution that is different from that of both Herschel starless and Herschel protostellar cores. While Giannini et al. (2012) found that the protostellar sources are quite compact (0.04-0.07 pc), in our submm sample protostellar cores are even less compact than starless ones.

\subsection{Core properties}

As can bee seen from Table 5, most of our CMF determinations are in very good agreement with a Salpeter IMF $(\alpha=-2.35)$.

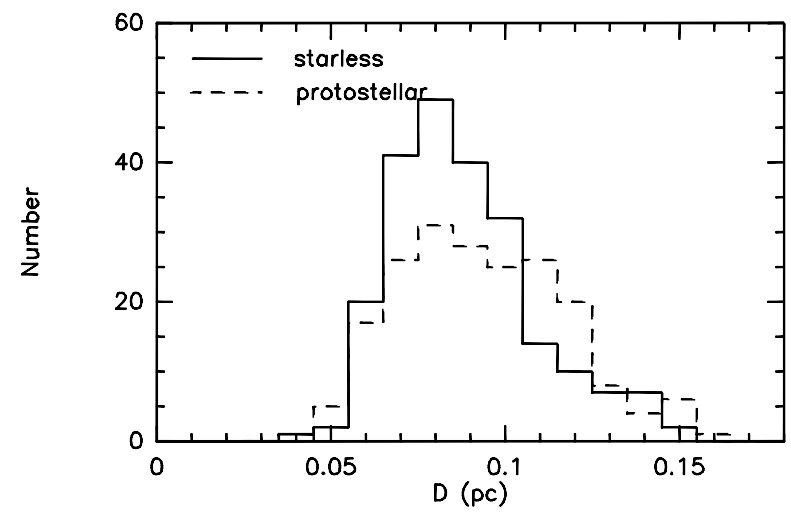

Fig. 17. Deconvolved size (diameter) distribution for starless (solid line) and protostellar (dashed line) submm cores. Only sources with minimum deconvolved size greater than half the beam FWHM have been taken into account.

This should be considered as a robust result, based on our discussion above. In fact, the ML estimator suggests a slightly flatter CMF for protostellar cores and a slightly steeper one for starless cores. As pointed out in Sect. 4.3, the starless CMF is actually representative of a real prestellar core population, so it can be compared with prestellar CMFs derived in other regions. Table 7 lists the results of a number of surveys carried out towards nearby star-forming regions. Prestellar CMFs of nearby regions (125 to $700 \mathrm{pc}$ ) obtained from single-dish observations with spatial resolutions in the range $0.01-0.07 \mathrm{pc}$, though sometimes shifted to higher masses, are all consistent with a Salpeter IMF in their high-mass end. Olmi et al. (2018) found that this similarity persists at a larger scale, deriving an average slope $\alpha=-2.12 \pm 0.15$ in a sample of clump mass functions extracted from the Hi-Gal catalogue. We note that in the most recent prescriptions the standard IMF is steeper than a Salpeter one; for example, Kroupa et al. (1993) propose $\alpha=-2.7$ for $M \geq 1 M_{\odot}$ whereas Scalo (1998) find $\alpha=-2.7 \pm 0.5$ in the range $1-10 M_{\odot}$. This agrees with the spectral index we found using the ML estimator for starless cores.

ALMA is now starting to address farther regions $(d>2 \mathrm{kpc})$ but with very good angular resolution. This is instrumental in probing different environments such as infrared dark clouds (IRDCs) and massive star-forming regions. Some prestellar CMFs recently derived from ALMA data are listed in Table 8. We note the cases for variations in the high-mass end of the CMF reported by Motte et al. (2018) and Liu et al. (2018). Both find top-heavy CMFs. However, these ALMA data are not easy to compare with single-dish data of nearby regions. As discussed by Liu et al. (2018), i) prestellar and protostellar cores cannot be separated given the larger distances, ii) lack of spatial frequencies has to be taken into account, and iii) the statistics are often poor. In addition, the statistics show a higher sensitivity to the adopted core-retrieval algorithms.

It is useful to study other global properties of the submm cores such as the mass-size relation. We derived a mass-size relation $M \sim D^{2.2}$ (see Sect. 4.2) for the whole (protostellar and starless) sample. Considering that the power-law index is sensitive to the core-retrieval algorithm $\left(M \sim D^{1.7}\right.$ when using the CLUMPFIND output) and cautioning against possible incompleteness effects, this is consistent with the mass-radius relation for clumps and molecular clouds $M \sim R^{1.9}$ found by Larson (1981). For the sake of comparison, the results of a few largescale surveys are listed in Table 9; all these studies suggest that molecular cores and clumps arrange their masses according to 
Table 7. CMF determinations in nearby star-forming regions.

\begin{tabular}{|c|c|c|c|c|}
\hline Region & $\begin{array}{l}\text { Distance } \\
\text { (pc) }\end{array}$ & Beam size & $\mathrm{CMF}^{(a)}$ & Reference \\
\hline Pipe Nebula & 160 & $\sim 0.03 \mathrm{pc}$ & Shifted IMF & Alves et al. (2007) \\
\hline Orion regions & $\sim 400$ & $14^{\prime \prime}$ at $850 \mu \mathrm{m}$ & Shifted IMF & Nutter \& Ward-Thompson (2007) \\
\hline Polaris Flare & 150 & $18^{\prime \prime}$ at $250 \mu \mathrm{m}$ & Shifted IMF & André et al. (2010) \\
\hline Aquila Rift & 260 & $18^{\prime \prime}$ at $250 \mu \mathrm{m}$ & $\alpha=-2.33 \pm 0.06$ & Könyves et al. (2015) \\
\hline Orion A & $\sim 400$ & $18^{\prime \prime}$ & $\alpha \sim-2.3$ & Ikeda et al. (2007) \\
\hline Perseus, Serpens, Ophiuchus & $125-260$ & $31^{\prime \prime}$ & $\alpha=-2.3 \pm 0.4$ & Enoch et al. (2008) \\
\hline Taurus L1495 cloud ${ }^{(b)}$ & 140 & $\sim 18^{\prime \prime}$ & $\mathrm{IMF}$ & Marsh et al. (2016) \\
\hline
\end{tabular}

Notes. ${ }^{(a)}$ Shifted IMF means that the whole CMF approximates a standard IMF when shifted to lower masses. ${ }^{(b)}$ Sources retrieved using the getsources algorithm of Men'shchikov et al. 2012.

Table 8. CMF determinations in farther star-forming regions.

\begin{tabular}{ccccccc}
\hline \hline Region & $\begin{array}{c}\text { Distance } \\
(\mathrm{kpc})\end{array}$ & Beam size & CMF & $\begin{array}{c}\text { Mass range } \\
\left(M_{\odot}\right)\end{array}$ & Algorithm & Reference \\
\hline IRDC G14.225-0.506 & $\sim 2$ & $\sim 3^{\prime \prime}$ & $\alpha=-2.6 \pm 0.7$ & $2.4-14$ & CLUMPFIND Ohashi et al. (2016) \\
G286.21+0.17 & $\sim 2.5$ & $\sim 1^{\prime \prime}$ & $\alpha=-2.24 \pm 0.17$ & $\sim_{1}$ & dendrogram Cheng et al. (2018) \\
G286.21+0.17 & $\sim 2.5$ & $\sim 1^{\prime \prime}$ & $\alpha=-1.64 \pm 0.13$ & $\sim_{1}$ & CLUMPFIND Cheng et al. (2018) \\
W43-MM1 & $\sim 5.5$ & $\sim 0^{\prime \prime} 44$ & $\alpha=-1.90 \pm 0.06,-1.96 \pm 0.02$ & $1.6-100$ & getsources & Motte et al. (2018) \\
7 IRDCs & $2.4-5.7$ & $\sim 1^{\prime \prime}$ & $\alpha=-1.70 \pm 0.13$ & $\geq 1.26$ & dendrogram & Liu et al. (2018) \\
\hline
\end{tabular}

Notes. ${ }^{(a)}$ References for core retrieving algorithms. CLUMPFIND: Williams et al. (1994); dendrogram: Rosolowsky et al. (2008); getsources: Men'shchikov et al. (2012).

Table 9. Mass-size relations $\left(M \sim D^{X}\right)$ from the literature.

\begin{tabular}{llll}
\hline \hline Sample & $X$ & Reference & Notes \\
\hline Clumps and molecular clouds & 1.9 & Larson (1981) & \\
Clumps and cores in Vela D & 1.74 & Massi et al. (2007) & (1) \\
Clumps and cores in Taurus & $\sim 1.9$ & Marsh et al. (2016) & \\
ATLASGAL sample of clumps & & & \\
associated with methanol masers & & & \\
YMOs, and HII regions & 1.67 & Urquhart et al. (2014) & (2) \\
Galactic clumps from MALT90 & 2.2 & Contreras et al. (2017) & (3) \\
\hline
\end{tabular}

Notes. (1) CLUMPFIND, adopting constant $T_{\text {dust }}=30 \mathrm{~K}$; (2) adopting constant $T_{\text {dust }}=20 \mathrm{~K}$; (3) $T_{\text {dust }}$ and column densities from Herschel and ATLASGAL data.

$M \propto D^{2}$, which is expected for ensembles of structures with the same column density over a large range of sizes.

A mass-size sequence, irrespective of the value of its powerlaw index, has important implications concerning the evolution of dense prestellar cores. Either these cores are quickly assembled, or they evolve inside the mass-size sequence itself. In the latter case, two scenarios can be envisaged. Simpson et al. (2011) suggested that cores are thermally supported and move along the mass-size sequence from the low-mass end, by accreting mass quasi-statically while maintaining a Bonnor-Ebert equilibrium. Once they reach Jeans instability, they collapse as protostars. Since starless cores in Vela $\mathrm{C}$ lie well above the mass instability limits, this model should be modified by introducing other forms of inner physical support (magnetic fields, turbulence). However, we note that the detection loci drawn in Fig. 7 would hide any signature of evolutionary paths from the critical BonnorEbert half-mass loci to the mass-size sequence. In addition, the adopted dust opacity affects the picture. In fact, using the ATLASGAL value would move the sequence and detection loci nearer to the Bonnor-Ebert loci. Nevertheless, studies of magnetic fields in Vela $\mathrm{C}$ based on FIR polarisation (Fissel et al. 2019) suggest a connection between large-scale magnetic fields and small gas structures, which deserves further investigation. Alternatively, the fact that a similar mass-size relation is found for clumps and cores may indicate that dense cores move down the sequence from the high-mass end by fragmentation (e.g. Elmegreen \& Falgarone 1996).

Hydrodynamic simulations can help us to understand dense core evolution as well. Li et al. (2004) performed turbulent simulations of a magnetised cloud, deriving both the global physical properties and the evolution of bound dense cores. Their CMF is consistent at the high-mass end with a power law of index $\alpha \sim-2.29$, but the mass-size relation is steeper than observed in Vela $\mathrm{C}\left(M \sim D^{2.7}\right)$. Interestingly, the CMF flattens with cloud age. Offner et al. (2008) simulated both driven and decaying turbulence in molecular clouds. They obtained bound cores exhibiting a CMF with a high-mass end consistent with a stellar IMF but a mass-size relation flatter than ours $(M \sim D)$. However, their statistics are poor and the core sizes they obtain are $<0.02 \mathrm{pc}$, less than our adopted spatial size limit. Offner \& Krumholz (2009) also analysed the shapes of cores from simulations with driven and decaying turbulence. The axis ratio distributions they obtained roughly resemble ours, with median and mean values in the range 0.6-0.7 and minimum values of $\sim 0.2$. Their size distributions cannot be compared with ours, which is biased by having excluded cores smaller than half the LABOCA beam size. However, prestellar and protostellar synthetic cores are smaller and distributed in a smaller range than those derived from Orion observations by Nutter \& Ward-Thompson (2007). Prestellar core formation has also been investigated by Gong \& Ostriker (2015) and Gong \& Ostriker (2011, 2009) for converging flows triggering star formation in the post-shock gas. In particular, by retrieving dense cores in snapshots of their simulations (which include turbulence and 
gravity with inflow Mach numbers in the range 2-16), Gong \& Ostriker (2015) can reproduce mass-size relations consistent with ours (e.g. those with inflow Mach numbers 4 and 8). Since their cores gain mass during their evolution, which occurs with lifetimes of $\sim 1 \mathrm{Myr}$, the observed mass-size sequence would enclose condensations in different stages of evolution. Notably, their simulations also reproduce a CMF which is consistent with a stellar IMF, although steeper in the high-mass end. By selecting all cores in the stage just before a sink particle is formed (i.e. on the verge of forming a protostar) they obtain both a shallower mass-size relation and a CMF with a steeper high-mass end, implying a significant deficit of high-mass cores.

Lee et al. (2017) applied the gravoturbulent fragmentation theory of Hennebelle \& Chabrier to a filamentary environment with magnetic fields, finding CMFs that resemble a Salpeter IMF at their high-mass end. Interestingly, these authors found that filaments with high mass per length $\left(>1000 M_{\odot} \mathrm{pc}^{-1}\right)$ are needed to produce massive cores. Otherwise, the CMF is steeper than a Salpeter IMF. Guszejnov \& Hopkins (2016) modelled the fragmentation of self-gravitating, turbulent molecular clouds from large to small scales semi-analytically, confirming that the CMF shape is similar to the IMF at the high-mass end.

Undoubtedly, all simulations point to turbulence and magnetic fields as important ingredients in shaping some of the statistical properties of prestellar cores in Vela $\mathrm{C}$, such as CMF and the mass-size relation. However, our observations do not capture which mechanisms are the dominating ones.

\subsection{Star formation activity in Vela $C$}

Vela $\mathrm{C}$ exhibits different levels of star formation activity in its various parts. The five regions identified by Hill et al. (2011) roughly contain the same mass, but the peak column density in the areas referred to as nests by Hill et al. (2011) and in the north is smaller than in the ridges $\left(\sim 1\right.$ vs. $\sim 2 \times 10^{23} \mathrm{~cm}^{-2}$; Hill et al. 2011). A large star cluster with massive stars and an HII region (RCW 36), and an embedded young cluster with intermediatemass stars (IRS 31) have already formed in the Centre-Ridge and in the north, respectively, whereas the South-Nest is the coldest region of Vela C (Hill et al. 2011).

As shown in Sect. 4.3, the fraction of protostellar cores varies along the cloud; it is smaller in the south and larger in the centre. If taken at face value, this would agree with a picture where the southern part of Vela $\mathrm{C}$ is the least evolved and the central part (hosting RCW 36) the most evolved. However, possible biases have to be taken into account. Baldeschi et al. (2017) studied how the physical parameters of detected compact sources are affected by degradation of spatial resolution. Using Herschel maps of nearby star-forming regions, they simulated the effect of an increasing distance. They clearly found a significant decrease in the fraction of prestellar cores and a consequent increase in the fraction of protostellar cores up to $1 \mathrm{kpc}$. Thus, an increasing number of prestellar cores is misidentified due to lack of spatial resolution. When prestellar cores lie in projection very near to a protostellar core, after degrading the spatial resolution they merge into one large protostellar core. A look at Fig. 14 shows that the most massive (and largest) cores were detected in the centre of Vela $\mathrm{C}$. This region is dominated by the CentreRidge, which hosts RCW 36. Minier et al. (2013) propose that RCW 36 formed in a sheet of gas roughly edge-on and is driving an expanding bubble of matter yielding an edge-on ring. This would explain the large maximum column density derived by Hill et al. (2011). If so, a significant degree of line-of-sight confusion could arise, leading to larger cores and an increased fraction of protostellar cores due to the effect envisaged by Baldeschi et al. (2017). This could also explain the slightly flatter CMF derived in the central (and northern) part of Vela $\mathrm{C}$ (see Fig. 14). Furthermore, the size distribution of both starless and protostellar cores (see Fig. 17) hints at a fraction of cores resulting from confusion of smaller condensations, which could explain why protostellar cores are less compact than starless ones. When a number of cores are merged into a single larger one by the finding algorithm, there is a high probability that one of them is a protostar, biasing the whole output as protostellar. Thus, larger cores are more likely to be labelled as protostellar. In this respect, it would be interesting to observe the largest cores with ALMA.

Notably, the CMF is slightly steeper in the southern part of Vela $\mathrm{C}$ both for starless and protostellar cores, although these are consistent with each other within $1 \sigma$ (Fig. 14 and Table 5). There is clearly a shortage of high-mass cores. This fact may further suggest that this is the least evolved area in Vela $\mathrm{C}$, where massive cores have not yet been formed. In turn, this might also indicate that low- and intermediate-mass stars have to form earlier than massive stars.

Based on our data, we propose that Vela $\mathrm{C}$ is composed of sheets of filaments with different orientations; we propose that nests are seen roughly face-on, and ridges are seen almost edgeon. The South-Nest is likely to be in an earlier phase of global contraction, whereas at least RCW 36 in the Centre-Ridge may originate from converging flows, explaining why this part of the cloud has already evolved into a massive star-forming region. This scenario would be consistent with observational studies of magnetic field orientation in the region (Kusune et al. 2016; Soler et al. 2017; Fissel et al. 2019), pointing to magnetic field enhancement by density increase in the Centre-Ridge (consistent with gas compression) and showing that the plane-of-the-sky magnetic field direction is chaotic in the South-Nest. In addition, Sano et al. (2018) suggest that RCW 36 formed $\sim 0.1$ Myr ago due to cloud-cloud collision.

In Sect. 4.6, we describe our investigation of how dense cores cluster. When using a spatial resolution of $\sim 1.8 \mathrm{pc}$, clusters of cores less than $2 \mathrm{pc}$ in size are found to be separated by projected distances in the range 2-6 pc. Multiple clusters of dense cores are retrieved inside each of the sub-regions identified by Hill et al. (2011) through a multiple-resolution analysis decomposition of Vela $\mathrm{C}$ at a scale of $2 \mathrm{pc}$ (see their Fig. 7). When using a spatial resolution of $\sim 0.9 \mathrm{pc}$ and counting starless and protostellar cores separately, partially segregated groups of starless and protostellar cores are retrieved with separations in the range 1-2 pc. These roughly correspond to the high column density regions $\left(>10^{22} \mathrm{~cm}^{-2}\right.$ ) identified by Hill et al. (2011). The core clustering is then a signature of gas fragmentation. In fact, these scale-lengths are roughly consistent both with a magnetised and a turbulent Jeans length for $B \sim 30 \mu \mathrm{G}$ or a velocity dispersion $\sim 2 \mathrm{~km} \mathrm{~s}^{-1}$ and a mean gas density $n_{\mathrm{H}_{2}}=10^{3} \mathrm{~cm}^{-3}$. Fissel et al. (2019) suggest that the magnetic field in Vela $\mathrm{C}$ must be globally at least trans-Alfvénic, hence important in the evolution of cloud structures. A simple Jeans thermal analysis is therefore not appropriate for Vela $\mathrm{C}$. Hierarchical core clustering and gas fragmentation down to scales of $\sim 0.1 \mathrm{pc}$ (i.e. less than the spatial resolution of our data) have been found in other nearby star-forming regions (e.g. Kainulainen et al. 2017; Mattern et al. 2018).

The star formation activity of Vela $\mathrm{C}$ can be qualitatively compared with that of Vela D (Massi et al. 2006, 2007; Elia et al. 2007; Olmi et al. 2009). Here, young embedded star clusters of $\sim 1 \mathrm{Myr}$ in age are located towards gas clumps inside filaments 
which outline the edges of expanding bubbles. This suggests that the newly born stars are actually a second-generation population and therefore that Vela D is more evolved than Vela C.

\section{Conclusions}

We studied the dust emission in a large-scale map of the GMC Vela C obtained at $345 \mathrm{GHz}(0.87 \mathrm{~mm})$ with LABOCA at the APEX submm telescope. Using the algorithm CuTEx, we decomposed the emission into 549 compact submm sources (plus another 15 associated with the HII region RCW 34), with sizes ranging from less than half the APEX beam $(0.03 \mathrm{pc})$ to $0.16 \mathrm{pc}$. The algorithm CLUMPFIND was also used for comparison purposes, retrieving 291 compact submm sources (plus another 104 with beam-convolved angular sizes smaller than the APEX beam size). CuTEx is more focused on detecting compact sources, so we have based our analysis on its output only. Here, the submm sources are referred to as dense cores, which is appropriate given the size range spanned. We used ancillary MIR (WISE) and FIR (Herschel) data to further separate starless and protostellar cores and derive core dust temperatures. Core masses have been computed assuming a dust opacity of $k_{v}(v=345 \mathrm{GHz})=0.86 \mathrm{~g}^{-1} \mathrm{~cm}^{2}$ (for consistency with Massi et al. 2007 and Olmi et al. 2009), a dust spectral index of $\beta=2$, and a gas-to-dust ratio of 100. Furthermore, we used Gaia DR2 data to test the distance to Vela C, obtaining a value of $950 \mathrm{pc}$, but we adopted $700 \mathrm{pc}$ for consistency with previous works, pending further analyses based on Gaia DR2 data. This does not affect our conclusions and in any case scaling factors to shift our results to $950 \mathrm{pc}$ are given in the appendix. Our main results are summarised as follows.

1. A comparison between the emission at $345 \mathrm{GHz}$ and that at $250 \mathrm{GHz}$ obtained from SIMBA observations in two subregions of Vela $C$, namely IRS 31 and RCW 36, shows that even where intense stellar feedback is expected, the bulk of submm emission is dominated by dust thermal emission at $10-30 \mathrm{~K}$ with an emissivity index in the range $\beta \sim 1-2$.

2. The dense cores were classified into protostellar (233 out of 549) and starless (316 out of 549) essentially on the basis of their association with red WISE/MSX point sources. We estimated that this allows the detection of protostars with luminosities down to $L_{\text {bol }} \sim 2-3 L_{\odot}$. Most of the starless submm cores lie in a region of the mass-size diagram where gravity dominates over thermal support, and are therefore likely to be prestellar in nature.

3. A dust temperature was assigned to the dense submm cores by positionally associating them with Herschel compact sources. Unfortunately, this only yielded dust temperatures for a subsample of 197 submm cores.

4. The dust temperature distribution is different for starless and protostellar cores, with starless cores constrained in the range of 9-12 $\mathrm{K}$ and protostellar cores peaking at $11 \mathrm{~K}$ with a tail at higher temperatures.

5. The core masses have been derived both for the subsample of cores associated with Herschel sources using the FIRderived temperatures (single-source temperature), and for the whole sample of submm cores by assuming a constant temperature $T_{\text {dust }}=12.5 \mathrm{~K}$. The latter approach allows for a more robust statistical analysis. Starless cores have median and average masses of $\sim 4-7 M_{\odot}$ and $\sim 6-10 M_{\odot}$, respectively, whereas protostellar cores have median and average masses of $\sim 8 M_{\odot}$ and $\sim 15 M_{\odot}$, respectively. Protostellar cores exhibit a size distribution which is less compact than that of prestellar cores.
6. A core mass function was derived and its high-mass end was fit by a $\mathrm{d} N / \mathrm{d} M \sim M^{\alpha}$ function. The power-law index $\alpha$ was also derived using the ML estimator, which is less prone to statistical bias. Assuming $T_{\text {dust }}=12.5 \mathrm{~K}$, we estimate a mass completeness limit of $\sim 3.7 M_{\odot}$. The effects of dust opacity, distance, and calibration errors and of the temperature are discussed in the text, leading to various determinations of $\alpha$. These are mostly consistent with a Salpeter IMF $(\alpha \sim-2.35)$. There are some indications of the protostellar CMF being slightly flatter than the starless CMF. This is probably due to temperature effects and/or source confusion in crowded areas. The ML estimator suggests that the starless CMF is actually slightly steeper than a Salpeter IMF $(\alpha=-2.8 \pm 0.2)$, consistent with more recent IMF determinations.

7. A comparison with theoretical works suggests that turbulence and magnetic field are likely to be instrumental in explaining core formation in Vela $\mathrm{C}$.

8. The dense cores tend to cluster into groups with distances in the range $1-6 \mathrm{pc}$, with hints of partial segregation of starless and protostellar cores. This probably reflects fragmentation of magnetised and/or turbulent clumps with density $\sim 10^{3} \mathrm{~cm}^{-3}$.

9. There are strong indications of different levels of star formation activity in different parts of Vela C. The southern part of the cloud hosts the lowest fraction of protostellar cores ( $\sim 35 \%$ of the submm dense cores), whereas the central part hosts the largest fraction $(\sim 52 \%)$. The most massive cores are found in the centre, where massive star formation is in progress in RCW 36. However, line-of-sight effects with core merging are expected to be more frequent in the centre of the cloud.

10. We propose that dense cores are distributed in filaments roughly contained in sheets, which are seen almost face on in the case of the nests and edge-on in the case of the ridges. Massive star formation in the Centre-Ridge may have been triggered by converging flows.

Acknowledgements. This paper is based on data acquired with the Atacama Pathfinder EXperiment (APEX). APEX is a collaboration between the Max Planck Institute for Radioastronomy, the European Southern Observatory, and the Onsala Space Observatory. The authors acknowledge the help and the support from the APEX staff and the observers for their contribution in obtaining this dataset. This publication makes use of data products from the Wide-field Infrared Survey Explorer, which is a joint project of the University of California, Los Angeles, and the Jet Propulsion Laboratory/California Institute of Technology, funded by the National Aeronautics and Space Administration. This work is also based in part on observations made with the Spitzer Space Telescope, which is operated by the Jet Propulsion Laboratory, California Institute of Technology under a contract with NASA. This research has also made use of the NASA/IPAC Infrared Science Archive, which is operated by the Jet Propulsion Laboratory, California Institute of Technology, under contract with the National Aeronautics and Space Administration. This work has made use of data from the European Space Agency (ESA) mission Gaia (https: //www. cosmos.esa.int/gaia), processed by the Gaia Data Processing and Analysis Consortium (DPAC, https://www.cosmos.esa.int/web/ gaia/dpac/consortium). Funding for the DPAC has been provided by national institutions, in particular the institutions participating in the Gaia Multilateral Agreement. This research makes also use of data products from the Midcourse Space Experiment. Processing of the data was funded by the Ballistic Missile Defense Organization with additional support from NASA Office of Space Science. T.Cs. acknowledges support from the Deutsche Forschungsgemeinschaft, DFG via the SPP (priority programme) 1573 "Physics of the ISM".

\section{References}

Alves, J., Lombardi, M., \& Lada, C. J. 2007, A\&A, 462, L17 André, Ph., Men'shchikov, A., Bontemps, S., et al. 2010, A\&A, 518, L102 
André, Ph., di Francesco, J., Ward-Thompson, D., et al. 2014, Protostars and Planets VI, eds. H. Beuther, R. S. Klessen, C. P. Dullemond, \& T. Henning, (Tucson: University of Arizona press), 27

Bailer-Jones, C. A. L. 2015, PASP, 127, 994

Baldeschi, A., Elia, D., Molinari, S., et al. 2017, MNRAS, 466, 3682

Benjamin, R. A., Churchwell, E., Babler, B. L., et al. 2003, PASP, 115, 953

Bergin, E. A., \& Tafalla, M. 2007, ARA\&A, 45, 339

Bik, A., Puga, E., Waters, L. B. F. M., et al. 2010, ApJ, 713, 883

Bronfman, L., Nyman, L.-A., \& May, J. 1996, A\&AS, 115, 81

Chabrier, G. 2003, PASP, 115, 763

Cheng, Y., Tan, J. C., Liu, M., et al. 2018, ApJ, 853, 160

Churchwell, E., Babler, B. L., Meade, M. R., et al. 2009, PASP, 121, 213

Contreras, Y., Rathborne, J. M., Guzman, A., et al. 2017, MNRAS, 466, 340

Csengeri, T., Weiss, A., Wyrowski, F., et al. 2016, A\&A, 585, A104

Csengeri, T., Bontemps, S., Wyrowski, F., et al. 2017, A\&A, 600, L10

Curtis, E. I., \& Richer, J. S. 2010, MNRAS, 402, 603

De Luca, M., Giannini, T., Lorenzetti, D., et al. 2007, A\&A, 474, 863

Dunham, M. M., Crapsi, A., Evans, N. J., II, et al. 2008, ApJS, 179, 249

Elia, D., \& Pezzuto, S. 2016, MNRAS, 461, 1328

Elia, D., Massi, F., Strafella, F., et al. 2007, ApJ, 655, 316

Ellerbroek, L. E., Bik, A., Kaper, L., et al. 2013, A\&A, 558, A102

Elmegreen, B. G., \& Falgarone, E. 1996, ApJ, 471, 816

Enoch, M. L., Glenn, J., Evans, N. J., et al. 2007, ApJ, 666, 982

Enoch, M. L., Evans, N. J., Sargent, A. I., et al. 2008, ApJ, 684, 1240

Fissel, L. M., Ade, P. A. R., Angilè, F. E., et al. 2016, ApJ, 824, 134

Fissel, L. M., Ade, P. A. R., Angilè, F. E., et al. 2019, ApJ, 878, 110

Gaia Collaboration (Prusti, T., et al.) 2016, A\&A 595, A1

Gaia Collaboration (Brown, A. G. A., et al.) 2018, A\&A, 616, A1

Giannini, T., Elia, D., Lorenzetti, D., et al. 2012, A\&A, 539, A156

Gong, H., \& Ostriker, E. C. 2009, ApJ, 699, 230

Gong, H., \& Ostriker, E. C. 2011, ApJ, 729, 120

Gong, M., \& Ostriker, E. C. 2015, ApJ, 806, 31

Griffith, M. R., \& Wright, A. E. 1993, AJ, 105, 1666

Güsten, R., Nyman, L. ̊, Schilke, P., et al. 2006, A\&A, 454, L13

Guszejnov, D., \& Hopkins, P. F. 2016, MNRAS, 459, 9

Hildebrand, R. H. 1983, QJRAS, 24, 267

Hill, T., Motte, F., Didelon, P., et al. 2011, A\&A, 533, A94

Hou, L. G., \& Han, J. L. 2015, MNRAS, 454, 626

Ikeda, N., Sunada, K., \& Kitamura, Y. 2007, ApJ, 665, 1194

Kainulainen, J., Stutz, A. M., Stanke, T., et al. 2017, A\&A, 600, A141

Koenig, X. P., Leisawitz, D. T., Benford, D. J., et al. 2012, ApJ, 744, 130

Könyves, V., André, Ph., Men'shchikov, A., et al. 2015, A\&A, 584, A91

Kroupa, P., Tout, C. A., \& Gilmore, G. 1993, MNRAS, 262, 545

Kryukova, E., Megeath, S. T., Gutermuth, R. A., et al. 2012, AJ, 144, 31

Kusune, T., Sugitani, K., Nakamura, F., et al. 2016, ApJ, 830, L23

Larson R. B. 1981, MNRAS, 194, 809

Lee, Y.-N., Hennebelle, P., \& Chabrier, G. 2017, ApJ, 847, 114

Li, P. S., Norman, M. L., Mac Low, M. M., \& Heitsch, F. 2004, ApJ, 605 800

Liseau, R., Lorenzetti, D., Nisini, B., Spinoglio, L., \& Moneti, A. 1992, A\&A, 265,577

Liu, M., Tan, J. C., Cheng, Y., \& Kong, S. 2018, ApJ, 862, 105
Lorenzetti, D., Spinoglio, L., \& Liseau, R. 1993, A\&A, 275, 489

Marsh, K. A., Kirk, J. M., André, Ph., et al. 2016, MNRAS, 459, 342

Martin, P. G., Roy, A., Bontemps, S., et al. 2012, ApJ, 751, 28

Maschberger, T., \& Kroupa, P. 2009, MNRAS, 395, 931

Massi, F., Giannini, T., Lorenzetti, D., et al. 1999, A\&AS, 136, 471

Massi, F., Lorenzetti, D., Giannini, T., \& Vitali, F. 2000, A\&A, 353, 598

Massi, F., Lorenzetti, D., \& Giannini, T. 2003, A\&A, 399, 147

Massi, F., Testi, L., \& Vanzi, L. 2006, A\&A, 448, 1007

Massi, F., De Luca, M., Elia, D., et al. 2007, A\&A, 466, 1013

Mattern, M., Kainulainen, J., Zhang, M., \& Beuther, H. 2018, A\&A, 616, A78

Men'shchikov, A., André, Ph., Didelon, P., et al. 2012, A\&A, 542, A81

Minier, V., Tremblin, P., Hill, T., et al. 2013, A\&A, 550, A50

Molinari, S., Schisano, E., Faustini, F., et al. 2011, A\&A, 530, A133

Motte, F., André, Ph., \& Neri, R. 1998, A\&A, 336, 150

Motte, F., Zavagno, A., Bontemps, S., et al. 2010, A\&A, 518, L77

Motte, F., Nony, T., Louvet, F., et al. 2018, Nat. Astron., 2, 478

Murphy, D. C., \& May, J. 1991, A\&A, 247, 202

Netterfield, C. B., Ade, P. A. R., Bock, J. J., et al. 2009, ApJ, 707, 1824

Nutter, D., \& Ward-Thompson, D. 2007, MNRAS, 374, 1413

Nyman, L. A.., Lerner, M., Nielbock, M., et al. 2001, ESO Mess., 106, 40

Offner, S. S. R., \& Krumholz, M. R. 2009, ApJ, 693, 914

Offner, S. S. R., Klein, R. I., \& McKee, C. F. 2008, ApJ, 686, 1174

Ohashi, S., Sanhueza, P., Chen, H.-R. V., et al. 2016, ApJ, 833, 209

Olmi, L., Ade, P. A. R., Anglés-Alcázar, D., et al. 2009, ApJ, 707, 1836

Olmi, L., Elia, D., Schisano, E., \& Molinari, S. 2018, MNRAS, 480, 1831

Ossenkopf, V., \& Henning, Th. 1994, A\&A, 291, 943

Padoan, P., Federrath, C., Chabrier, G., et al. 2014, Protostar and Planet VI, eds. H. Beuther, R. S. Klessen, C. P. Dullemond, \& Th. Henning (Tucson: University of Arizona Press), 77

Palla, F., \& Stahler, S. W. 1993, ApJ, 418, 414

Rosolowsky, E. W., Pineda, J. E., Kauffmann, J., \& Goodman, A. A. 2008, ApJ, 679,1338

Salpeter, E. E. 1955, ApJ, 121, 161

Sano, H., Enokija, R., Hayashi, K., et al. 2018, PASJ, 70, S43

Scalo, J. 1998, The Stellar Initial Mass Function, Proceedings of the 38th Herstmonceux Conference, eds. G. Gilmore \& D. Howell, ASP Conf. Ser., 142, 201

Schnee, S., Mason, B., Di Francesco, J., et al. 2014, MNRAS, 444, 2303

Schneider, N., \& Brooks, K. 2004, PASA, 21, 290

Schuller, F. 2012, SPIE Conf. Ser., 8452, 84521T

Schuller, F., Menten, K. M., Contreras, Y., et al. 2009, A\&A, 504, 415

Simpson, R. J., Johnstone, D., Nutter, D., Ward-Thompson, D., \& Whitworth, A. P. 2011, MNRAS, 417, 216

Siringo, G., Kreysa, E., Kovács, A., et al. 2009, A\&A, 497, 945

Soler, J. D., Ade, P. A. R., Angilè, F. E., et al. 2017, A\&A, 603, A64

Strafella, F., Lorenzetti, D., Giannini, T., et al. 2015, ApJ, 798, 104

Urquhart, J. S., Moore, T. J. T., Csengeri, T., et al. 2014, MNRAS, 443, 1555

Whitney, B. A., Indebetouw, R., Bjorkman, J. E., \& Wood, K. 2004, ApJ, 617, 1177

Williams, J. P., de Geus, E. J., \& Blitz, L. 1994, ApJ, 428, 693

Wright, E. L., Eisenhardt, P. R. M., Mainzer, A. K., et al. 2010, AJ, 140, 1868

Yamaguchi, N., Mizuno, N., Saito, H., et al. 1999, PASJ, 51, 775 
Appendix A: Distance to Vela C

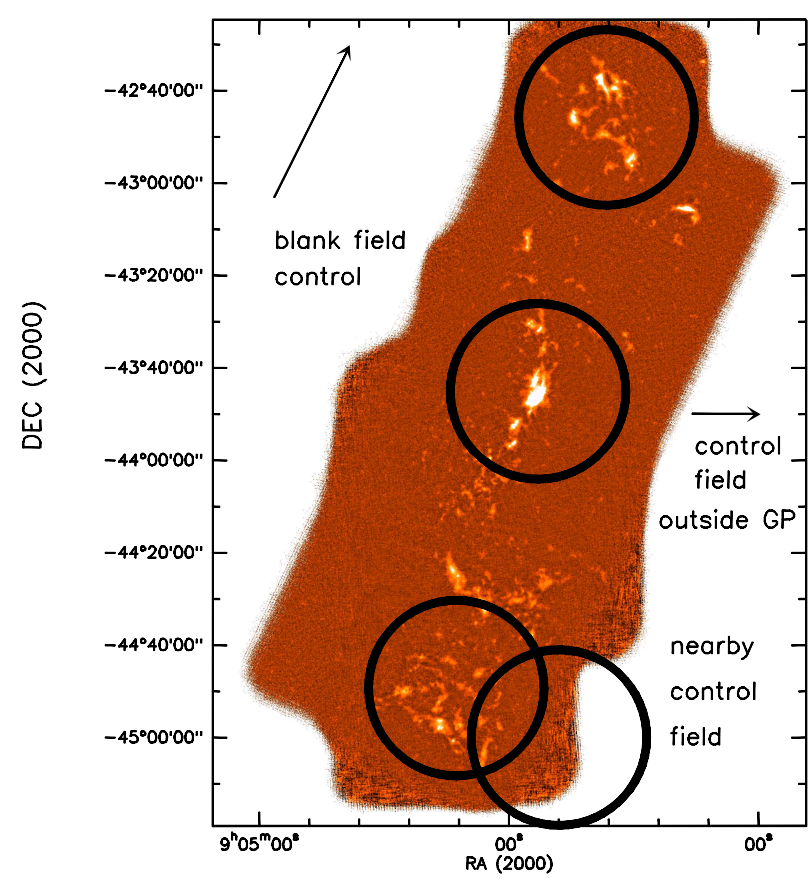

Fig. A.1. Selected fields overlaid on the LABOCA map of Vela C.

Stellar parallaxes from Gaia DR2 (Gaia Collaboration 2016, 2018) can be used to test the distance to Vela C. In this respect, we are interested in checking that Vela $\mathrm{C}$ lies in the range of values derived by Liseau et al. (1992) rather than revise it. Thus, we retrieved stellar parallaxes from the Gaia archive ${ }^{4}$ in a few circular fields of $20^{\prime}$ in radius, so that they roughly span the width of the molecular gas distribution when centred inside Vela $\mathrm{C}$. We selected sources with a parallax-to-error ratio $\geq 5$ to avoid the bias effects discussed in Bailer-Jones (2015). We selected three fields inside Vela C (see Fig. A.1), one centred on sub-field North, one centred on RCW36, and one centred on the South-Nest. In addition, we selected three control fields, one located $\sim 0.3 \mathrm{~h}$ west of RCW36 (i.e. outside the Galactic plane), one located $\sim 0.003 \mathrm{~h}$ west of the South-Nest, and one located at $l=240^{\circ}, b=$ $0^{\circ}$. In particular, the latter lies inside the "blank field", which is an area in the Galactic Plane adjacent to the VMR but devoid of molecular emission (Lorenzetti et al. 1993). All parallaxes were converted into distances and counted inside bins of $50 \mathrm{pc}$ in width. The data are shown in Fig. A.2. The farther field outside the Galactic plane (Fig. A.2a) clearly shows that the number $N$ of stars increases steadily with distance $d$. The data have been locally fitted by $N \sim d^{0.7}$ (solid line in figure). As a first approximation, the same relation should hold towards Vela $\mathrm{C}$ provided it is multiplied by a constant $>1$ (to account for the increase in $N$ towards the Galactic Plane). In fact, when multiplied by 2 it roughly fits the nearest control field (histogram in Fig. A.2b, red line) up to $\sim 1000 \mathrm{pc}$, where the number of stars suddenly decreases. This should mark absorption from molecular gas associated with Vela $\mathrm{C}$, as the control field located in
Table A.1. Effects of distance on the derived parameters.

\begin{tabular}{ccc}
\hline \hline Parameter & At $700 \mathrm{pc}$ & At $950 \mathrm{pc}$ \\
\hline CMF slope & $\alpha$ & $\alpha$ \\
Log(size)- $\log ($ mass) slope & $x$ & $x$ \\
$\quad$ Sizes & $D$ & $1.36 \times D$ \\
$\begin{array}{c}\text { and linear resolution } \\
\text { Masses }\end{array}$ & $M$ & $1.84 \times M$ \\
$\begin{array}{c}\text { and mass completeness limit } \\
70 \mu \text { m sensitivity } \\
\text { to protostars }\end{array}$ & $0.28 L_{\odot}$ & $0.5 L_{\odot}$ \\
$\begin{array}{c}\text { WISE sensitivity } \\
\text { to protostars }\end{array}$ & $1.7-2.8 L_{\odot}$ & $3.1-5.0 L_{\odot}$ \\
$\begin{array}{c}\text { Bonnor-Ebert critical mass } \\
\text { as a function of } \text { apparent size }\end{array}$ & $0.4-0.5 M_{\odot}$ & $0.6-0.7 M_{\odot}$ \\
\hline
\end{tabular}

the blank field (blue histogram in Fig. A.2b), though not fitted by the $N-d^{0.7}$ relation, nevertheless exhibits a steady increase in $N$. One has to keep in mind that, unlike the blank field, the line of sight to Vela $\mathrm{C}$ is roughly tangential to the local arm (e.g. Hou \& Han 2015), which is not taken into account in our scaled $N-d$ relation.

The three fields inside Vela C (Figs. A.2c-e) clearly display an abrupt decrease in the number of stars between 900 and $1000 \mathrm{pc}$, like that in the nearest control field. By comparing with the farthest control field, we believe that this is caused by the shielding effect of the molecular gas. This means that stars behind the gas are so extincted that the number of stars bright enough to allow parallax determination decreases strongly. The Gaia data suggest a distance of $\sim 950 \pm 50 \mathrm{pc}$. Interestingly, a shift from 900 to $1000 \mathrm{pc}$ is evident from south to north, suggesting an extent of $\sim 100 \mathrm{pc}$ along the line of sight, roughly comparable to the projected length of Vela $\mathrm{C}(\sim 36 \mathrm{pc})$.

The fields towards RCW36 and the South-Nest also exhibit a plateau between 500 and $900 \mathrm{pc}$ which is barely consistent with Poisson fluctuations. As we are observing along the local arm, a superposition of local clouds and stellar groups is expected. To disentangle the different components and confirm the distance to Vela C, a study of stellar colours as a function of parallaxes is necessary, which is beyond the scope of this paper. Pending a careful analysis of the stellar populations in the line of sight towards Vela $\mathrm{C}$, and more accurate parallax determinations from the next Gaia releases, with the data at our disposal we can exclude the nearer distance interval derived by Liseau et al. (1992) and conclude that the region is located between 700 and $1000 \mathrm{pc}$, with $\sim 950 \pm 50 \mathrm{pc}$ as a likely value.

We note that the updated distance would not significantly change the results discussed in the paper. The slopes derived through linear fits would not change. The cores would be slightly larger and more massive, but this is not a problem, and the sensitivity to protostellar cores only slightly worse. The differences are listed in Table A.1. From Table A.1, it is clear that core masses in Fig. 7 are shifted vertically more than the loci of Bonnor-Ebert critical half-masses, so the issue of gravitational boundedness is reinforced if the updated distance is assumed.

\footnotetext{
4 https://archives.esac.esa.int/gaia
} 


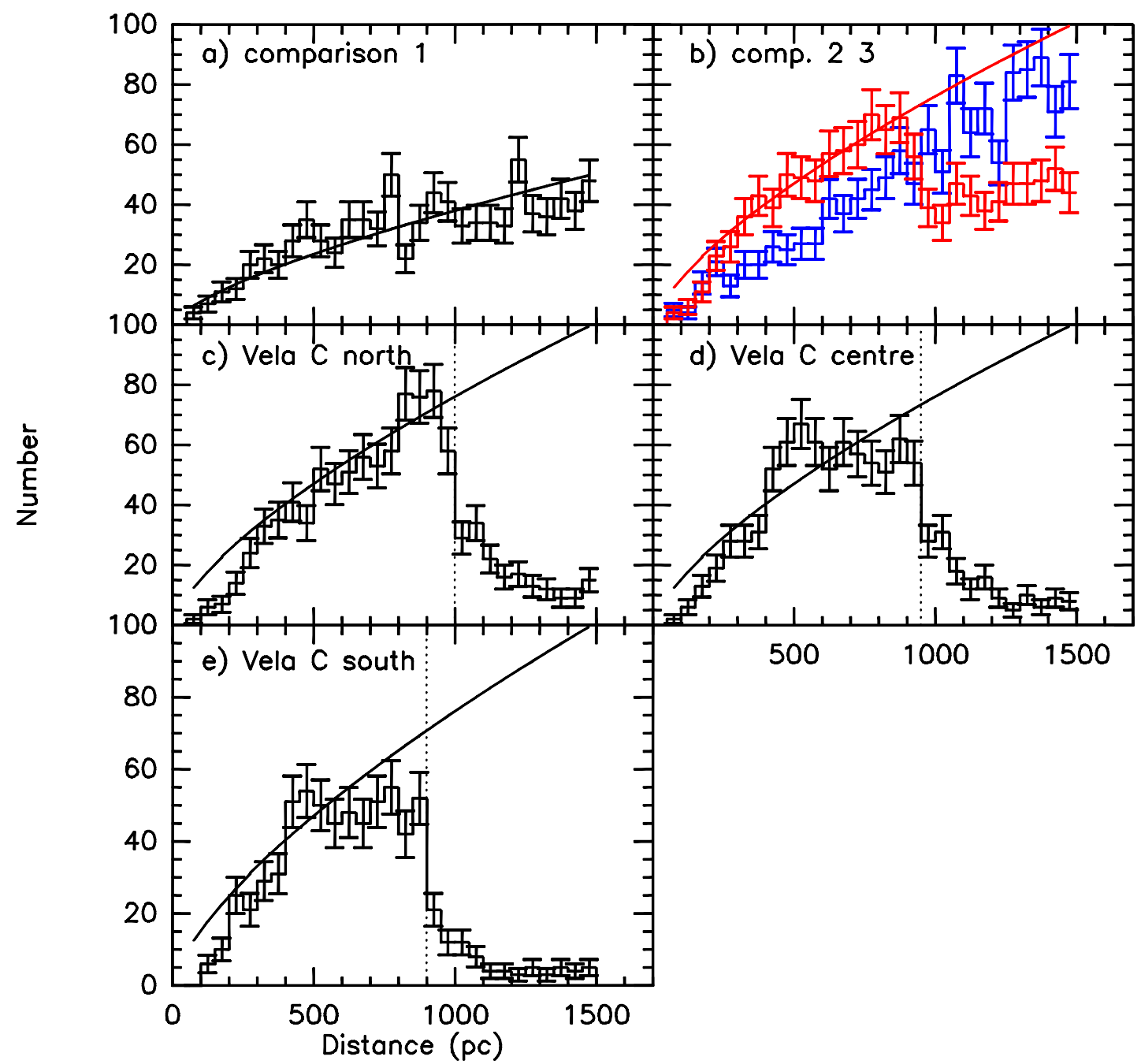

Fig. A.2. Distance of stars in the line of sight towards three Vela C fields and three control fields (see text) from Gaia DR2. The number of stars is displayed in bins of $50 \mathrm{pc}$ in width, error bars are computed according to Poissonian statistics. $a$ : farther control field outside the Galactic Plane, $b$ : nearer control field outside the Galactic Plane (red) and control field located at $l=240^{\circ}, b=0^{\circ}$ (blue), $c$ : field centred on sub-field North, $d$ : field centred on RCW36, and $e$ : field centred on the South-Nest. The full line is a $N \sim d^{0.7}$ fit to the data in $a$, multiplied by two in $b$ to $e$. The vertical dotted lines mark the sudden decrease in the number of stars, which is likely to coincide with the location of the molecular gas.

\section{Appendix B: Comparison of core masses towards RCW 36}

Minier et al. (2013) found seven clumps surrounding RCW 36 by combining FIR Herschel data and submm $(450 \mu \mathrm{m})$ groundbased data. All these structures are retrieved by CuTEx in our LABOCA map, but for only four we were able to assign a dust temperature from the association with the Herschel cores of Giannini et al. (2012). The masses computed from the submm emission mapped with LABOCA (see Table 1) by using these temperatures are compared with those computed by Minier et al. (2013) in Table B.1. Although the masses from our data are consistent with the ranges of values that they indicate for their sources 5 and 7, sources 1 and 3 are much less massive than we find. They adopted temperatures of $17-25 \mathrm{~K}$ to derive the ranges of masses, which are consistent with temperatures of 20-21 K found from the Herschel data. In addition, their adopted opacity would result in $1.2 \mathrm{~cm}^{2} \mathrm{~g}^{-1}$ at $345 \mathrm{GHz}$, as opposed to our adopted $0.86 \mathrm{~cm}^{2} \mathrm{~g}^{-1}$, that is only $30 \%$ more. From Table 2 of Minier et al. (2013), the highest masses are obtained by measuring the flux at $350 \mu \mathrm{m}$ in a circular aperture $25^{\prime \prime}$ in diameter. This is less than the size of the corresponding submm cores (see
Table B.1. Comparison of the masses of cores towards RCW 36 derived by Minier et al. (2013) and from our LABOCA data (Cols. 3-4).

\begin{tabular}{cccc}
\hline \hline $\begin{array}{c}\text { Source ID } \\
\text { in Minier et al. 2013 }\end{array}$ & Mass & $\begin{array}{c}\text { Source ID } \\
\text { in Table 1 }\end{array}$ & $\begin{array}{c}\text { Mass } \\
\left(M_{\odot}\right)\end{array}$ \\
\hline 1 & $17-72$ & 453 & 128 \\
3 & $4-25$ & 449 & 115 \\
5 & $10-25$ & 446 & 9 \\
7 & $3-19$ & 457 & 17 \\
\hline
\end{tabular}

Table 1). On the other hand, they find lower masses by combining fluxes at 160 and $450 \mu \mathrm{m}$ with a better spatial resolution $\left(12^{\prime \prime}\right)$, which suggests a higher degree of source confusion at lower spatial resolutions. In fact, in Sect. 5.3 we propose that source confusion leading to the merging of unresolved sources may yield the most massive cores in the centre region. This inconsistency highlights real differences arising from combining data at different wavelengths with different sensitivities and spatial resolutions. 